\title{
Robust Exponential Stabilization of Stochastic Delay Interval Recurrent Neural Networks with Distributed Parameters and Markovian Jumping by Using Periodically Intermittent Control
}

\author{
Junhao Hu, ${ }^{1}$ Yunjian Peng, ${ }^{2}$ and ${\text { Yan } \mathrm{Li}^{3,4}}^{3,4}$ \\ ${ }^{1}$ College of Mathematics and Statistics, South Central University for Nationalities, Wuhan 430074, China \\ ${ }^{2}$ School of Automation Science and Engineering, South China University of Technology, Guangzhou 510641, China \\ ${ }^{3}$ College of Automation, Huazhong University of Science and Technology, Wuhan 430074, China \\ ${ }^{4}$ College of Science, Huazhong Agriculture University, Wuhan 430070, China \\ Correspondence should be addressed to Yan Li; ly@mail.hzau.edu.cn
}

Received 13 January 2014; Accepted 14 February 2014; Published 27 April 2014

Academic Editor: Zhengguang Wu

Copyright (C) 2014 Junhao Hu et al. This is an open access article distributed under the Creative Commons Attribution License, which permits unrestricted use, distribution, and reproduction in any medium, provided the original work is properly cited.

\begin{abstract}
We consider a class of stochastic delay recurrent neural networks with distributed parameters and Markovian jumping. It is assumed that the coefficients in these neural networks belong to the interval matrices. Several sufficient conditions ensuring robust exponential stabilization are derived by using periodically intermittent control and Lyapunov functional. The obtained results are very easy to verify and implement, and improve the existing results. Finally, an example with numerical simulations is given to illustrate the presented criteria.
\end{abstract}

\section{Introduction}

In recent decades, neural network dynamics has been widely studied by many authors due to the fact that neural network dynamics can be applied to associate memory, signal processing, pattern classification, and quadratic optimization. Liao and Mao $[1,2]$ investigated the stability of stochastic neural network for the first time in 1996. By Razumikhintype theorems, the stability of stochastic neural networks with variable delays was considered [3]. Considering electrons moving in the asymptotic electromagnetic field, the diffusion phenomena could not be ignored. Luo et al. [4] gave several algebra criteria for stochastic Hopfield neural networks with distributed parameters by using average Lyapunov function. The asymptotic stability of stochastic reaction- diffusion systems was also established in [5]. The asymptotic behavior of several classes of neural networks with reaction-diffusion terms has been reported in [6-9]. Hu et al. [10] discussed the exponential stability and synchronization of delay neural networks with reaction-diffusion terms by impulsive control.

However, the parameters in neural networks are always some uncertainty and error. Taking these uncertainty and error into account, $\mathrm{Xu}$ et al. [11] investigated stochastic exponential robust stability of interval neural networks with reaction-diffusion terms and mixed delays by applying the vector Lyapunov function method and $M$-matrix theory. Wang and Gao [12] studied global exponential robust stability of reaction-diffusion interval neural networks with timevarying delays by means of the topological degree theory and Lyapunov functional method. And, a sufficient condition was presented for robust global exponential stability of interval reaction-diffusion Hopfield neural networks with distributed delays by constructing Lyapunov functional and utilizing some inequality techniques [13].

The neural networks driven by continuous-time Markov Chains have been also used to model many practical neural networks because they may experience abrupt changes in their structure and parameters caused by phenomena such as component failures or repairs, changing subsystem interconnections, and abrupt environmental disturbances. The exponential stability and stabilization of recurrent neural networks with Markovian jumping were discussed in [1420]. Robust stability of stochastic delayed additive neural networks with Markov jumping was investigated in [21]. Mao 
[22] studied the stability of stochastic delay interval system with Markovian jumping by linear matrix inequality.

Many control approaches have been developed to stable and synchronized system such as impulsive control [23] and intermittent control [24-29]. Gan [24-26] revealed exponential synchronization of three classes of stochastic delay neural networks via periodically intermittent control. $\mathrm{Hu}$ et al. [27, 28] investigated exponential stabilization and synchronization of delay neural networks. Huang et al. [29] studied stabilization of delayed chaotic neural networks by periodically intermittent control.

In this paper, we will consider a class of stochastic delay interval recurrent neural networks with distributed parameters and Markovian switching whose active functions are more general than the Lipschitz continuous active function [24-26] and the monotone active function [2729]. By the average Lyapunov functional and periodically intermittent control, several sufficient conditions ensuring robust exponential stabilization are given. Therefore, the organization of this paper is as follows. Some preliminaries and introduction are given in Section 2. In Section 3, robust exponential stabilization of these stochastic neural networks is proved. An example with numerical simulation is given to illustrate the effectiveness of the obtained results in Section 4.

\section{Preliminaries}

Throughout this paper, unless otherwise specified, we let $\left(\Omega, \mathscr{F},\left\{\mathscr{F}_{t}\right\}_{t \geq 0}, \mathbb{P}\right)$ be a complete probability space with a filtration $\{\mathscr{F}\}_{t \geq 0}$ satisfying the usual conditions (i.e., it is right-continuous and $\mathscr{F}_{0}$ contains all $\mathbb{P}$-null sets). Let $\mathbb{R}^{m}$ be the $m$-dimensional Euclidean space and let $|\cdot|$ be the Euclidean norm in $\mathbb{R}^{m}, \mathbb{R}_{+}=[0,+\infty)$ and $\tau>0$. Assuming that $\Omega_{0} \subset \mathbb{R}^{m}$ is a bounded compact set with smooth boundary $\partial \Omega_{0}$ and mes $\Omega_{0}>0$ in space $\mathbb{R}^{m}$. Let $C([-\tau, 0] \times$ $\left.\Omega_{0} ; \mathbb{R}^{n}\right)$ denote the family of continuous function $\phi(t, x)$ from $[-\tau, 0] \times \Omega_{0}$ to $\mathbb{R}^{n}$ with $\|\phi\|=\sup _{-\tau \leq t \leq 0, x \in \Omega_{0}}|\phi(t, x)|$. Denote by $C_{\mathscr{F}_{0}}^{b}\left([-\tau, 0] \times \Omega_{0}, \mathbb{R}^{n}\right)$ the family of all bounded, $\mathscr{F}_{0^{-}}$ measurable, $C\left([-\tau, 0] \times \Omega_{0} ; \mathbb{R}^{n}\right)$-valued random variables. Let $W(t), t \geq 0$ be $n$-dimension Brownian motion defined on the probability space. Let $r(t), t \geq 0$ be right-continuous Markov chain on the probability space taking values in a finite state space $\mathbb{S}=\{1,2, \ldots, N\}$ with generator $\Gamma=\left(r_{i j}\right)_{N \times N}$ given by

$$
\begin{aligned}
& \mathbb{P}\{r(t+\Delta)=j \mid r(t)=i\} \\
& \quad= \begin{cases}\gamma_{i j} \Delta+o(\Delta) & \text { if } i \neq j \\
1+\gamma_{i i} \Delta+o(\Delta) & \text { if } i=j,\end{cases}
\end{aligned}
$$

where $\Delta>0$. Here, $r_{i j} \geq 0$ is the transition rate from $i$ to $j$ if $i \neq j$ while

$$
\gamma_{i i}=-\sum_{j \neq i} \gamma_{i j}
$$

We assume that the Markov chain $r(\cdot)$ is independent of the Brownian motion $W(\cdot)$. It is well known that almost every sample path of $r(t)$ is right-continuous step function with a finite number of simple jumps in any finite subinterval $\mathbb{R}_{+}$.
In this paper, we consider a class of stochastic delay interval recurrent neural networks with distributed parameters and Markovian jumping:

$$
\begin{aligned}
& d u_{i}(t, x)=\left\{\sum_{k=1}^{m} \frac{\partial}{\partial x_{k}}\left(D_{i k}(r(t)) \frac{\partial u_{i}(t, x)}{\partial x_{k}}\right)\right. \\
&-a_{i}(r(t)) u_{i}(t, x) \\
&+\sum_{j=1}^{n} b_{i j}(r(t)) f_{j}\left(u_{j}(t, x)\right) \\
&\left.+\sum_{j=1}^{n} c_{i j}(r(t)) g_{j}\left(u_{j}\left(t-\tau_{i j}(t)\right), x\right)\right\} d t \\
&+\sum_{j=1}^{n} h_{i j}\left(u_{j}(t, x), u_{j}\left(t-\tau_{i j}(t), x\right)\right) d W_{j}(t),
\end{aligned}
$$

for $t \geq 0, i=1,2, \ldots, n$, where $n \geq 2$ denotes the number of neurons in neural networks. $x=\left(x_{1}, x_{2}, \ldots, x_{m}\right)^{T} \in \Omega_{0} \subset$ $\mathbb{R}^{m}$ is the space variable, $\Omega_{0}=\left\{x=\left(x_{1}, x_{2}, \ldots, x_{m}\right)^{T}|| x_{k} \mid<\right.$ $\left.\theta_{k}, k=1,2, \ldots, m\right\}$ is a bounded compact set with smooth boundary $\partial \Omega_{0}$, and mes $\Omega_{0}>0$ in space $\mathbb{R}^{m}$. $u(t, x)=\left(u_{1}(t, x), u_{2}(t, x), \ldots, u_{n}(t, x)\right)^{T}$ corresponds to the state variable of the $i$ th neural in space $x$ and at time $t$. $D_{i k}(r(t)) \geq 0$ denotes the transmission diffusion operator along the $i$ th neuron. $a_{i}(r(t))>0$ denotes the changing time constant or passive decay rate of the $i$ th neuron. $b_{i j}(r(t))$ and $c_{i j}(r(t))$ denote the connection weight and the delayed connection weight of the $j$ th neuron on the $i$ th neuron, respectively. $\tau_{i j}(t)$ corresponds to the transmission delay and satisfies $0 \leq \tau_{i j}(t) \leq \tau, \dot{\tau}_{i j}(t) \leq \tau_{0}<1$ for all $t \geq 0\left(\tau, \tau_{0}\right.$ is a constant, $\left.i, j=1,2, \ldots, n\right) . h_{i j}(\cdot, \cdot)$ denotes stochastic perturbation function to the neuron.

The boundary condition of system (3),

$$
\left.u(t, x)\right|_{\partial \Omega_{0}}=0, \quad(t, x) \in[-\tau,+\infty) \times \partial \Omega_{0}, i=1,2, \ldots, n .
$$

The initial value of system (3),

$$
u(t, x)=\phi_{i}(t, x), \quad(t, x) \in[-\tau, 0) \times \Omega_{0}, i=1,2, \ldots, n .
$$

Moreover, $A(r(t))=\operatorname{diag}\left(a_{1}(r(t)), \ldots, a_{n}(r(t))\right), B(r(t))=$ $\left(b_{i j}(r(t))\right)_{n \times n}$, and $C(r(t))=\left(c_{i j}(r(t))\right)_{n \times n}$ are the interval connection weight matrix for each value of $r(t)$ in $\mathbb{S}$ with the initial value $r(0)=r_{0} ; D(r(t))=\left(b_{i j}(r(t))\right)_{n \times m}$ is interval transmission diffusion operator matrix for each value of $r(t)$ in $\mathbb{S}$ with the initial value $r(0)=r_{0}$. 
For convenience, we give the following notions that for $r(t)=l$ in $\mathbb{S}$ :

$$
\begin{gathered}
A^{*}=\left\{A(l)=\operatorname{diag}\left(a_{1}(l), \ldots, a_{n}(l)\right): \underline{A}(l) \leq A(l) \leq \bar{A}(l),\right. \\
\text { i.e., } \left.\underline{a}_{i}(l) \leq a_{i}(l) \leq \bar{a}_{i}(l), i=1,2, \ldots, n\right\} ; \\
B^{*}=\left\{B(l)=\left(b_{i j}(l)\right)_{n \times n}: \underline{B}(l) \leq B(l) \leq \bar{B}(l),\right. \\
\text { i.e., } \left.\underline{b}_{i j}(l) \leq b_{i j}(l) \leq \bar{b}_{i j}(l), i, j=1,2, \ldots, n\right\} ; \\
C^{*}=\left\{C(l)=\left(c_{i j}(l)\right)_{n \times n}: \underline{C}(l) \leq C(l) \leq \bar{C}(l),\right. \\
\text { i.e., } \left.\underline{c}_{i j}(l) \leq c_{i j}(l) \leq \bar{c}_{i j}(l), i, j=1,2, \ldots, n\right\} ; \\
D^{*}=\left\{D(l)=\left(d_{i j}(l)\right)_{n \times n}: \underline{D}(l) \leq D(l) \leq \bar{D}(l),\right. \\
\text { i.e., } \underline{d}_{i j}(l) \leq d_{i j}(l) \leq \bar{d}_{i j}(l), \\
i=1,2, \ldots, n, j=1,2, \ldots, m\} .
\end{gathered}
$$

Definition 1. The stochastic vector $u(t, x)=\left(u_{1}(t, x), u_{2}(t, x)\right.$, $\left.\ldots, u_{n}(t, x)\right)^{T}$ is called the solution of system (3)-(5), if it satisfies the following conditions:

(i) $u(t, x)$ is adapted to $\left\{\mathscr{F}_{t}\right\}_{t \geq 0}$;

(ii) for every $T_{0} \in \mathbb{R}_{+}, u(t, x) \in C_{\mathscr{F}_{0}}^{b}\left(\left[0, T_{0}\right] \times \Omega_{0} ; \mathbb{R}^{n}\right)$ and

$$
\mathbb{E}\left(\max _{x \in \Omega_{0}} \int_{0}^{T_{0}}\left[|u(t, x)|^{2}+|\nabla u(t, x)|^{2}\right] d t\right)<+\infty
$$

(iii) for every $t \in \mathbb{R}_{+}$,

$$
\begin{aligned}
& \int_{\Omega_{0}} u_{i}(t, x) d x \\
& =\int_{\Omega_{0}} \phi_{i}(0, x) d x+\int_{\Omega_{0}} \int_{0}^{t} \sum_{k=1}^{m} \frac{\partial}{\partial x_{k}} \\
& \quad \times\left(D_{i k}(r(s)) \frac{\partial u_{i}(s, x)}{\partial x_{k}}\right) d s d x \\
& +\int_{\Omega_{0}} \int_{0}^{t}\left[-a_{i}(r(s)) u_{i}(s, x)+\sum_{j=1}^{n} b_{i j}(r(s)) f_{j}\left(u_{j}(s, x)\right)\right. \\
& +\int_{\Omega_{0}} \int_{0}^{t} \sum_{j=1}^{n} h_{i j}\left(u_{j}(s, x), u_{j}\left(s-\tau_{i j}(s), x\right)\right) d W_{j}(s) d x, \\
& \quad(t, x) \in\left[0, T_{0}\right] \times \Omega_{0},
\end{aligned}
$$

so it holds as $\mathbb{P}$-a.s., $i=1,2, \ldots, n$.
Definition 2. System (3)-(5) is called robust exponential stable in $p$ th moment for any $A(l) \in A^{*}, B(l) \in B^{*}, C(l) \in C^{*}$, $D(l) \in D^{*}, l \in \mathbb{S}$ if the solution $u(t, x)$ of system (3)-(5) satisfies

$$
\limsup _{t \rightarrow+\infty} \frac{1}{t} \log \left(\mathbb{E}\|u(t, x)\|^{p}\right)<0
$$

where $\|u(t, x)\|=\left(\int_{\Omega_{0}}|u(t, x)|^{p} d x\right)^{1 / p},(t, x) \in \mathbb{R}_{+} \times \Omega_{0}$.

To assure the existence and uniqueness of the solution to system (3)-(5) (see, [30, 31]), we give the following assumptions:

(H1) for $i=1,2, \ldots n, \forall s_{1}, s_{2} \in \mathbb{R}$, the neuron activation functions $f_{i}, g_{i}$ are bounded, $f_{i}(0)=g_{i}(0)=0$, and satisfy

$$
\begin{aligned}
& L_{i}^{-} \leq \frac{f_{i}\left(s_{1}\right)-f_{i}\left(s_{2}\right)}{s_{1}-s_{2}} \leq L_{i}^{+}, \\
& N_{i}^{-} \leq \frac{g_{i}\left(s_{1}\right)-g_{i}\left(s_{2}\right)}{s_{1}-s_{2}} \leq N_{i}^{+},
\end{aligned}
$$

where $s_{1} \neq s_{2}$, and $L_{i}^{-}, L_{i}^{+}, N_{i}^{-}, N_{i}^{+}$are constants.

(H2) For $i, j=1,2, \ldots, n, \forall s_{1}, s_{2}, \widetilde{s}_{1}, \widetilde{s}_{2} \in \mathbb{R}$, there exists positive constant $\sigma_{i j}$, such that

$$
\left|h_{i j}\left(s_{1}, s_{2}\right)-h_{i j}\left(\widetilde{s}_{1}, \widetilde{s}_{2}\right)\right|^{2} \leq \sigma_{i j}\left(\left|s_{1}-\widetilde{s}_{1}\right|^{2}+\left|s_{2}-\widetilde{s}_{2}\right|^{2}\right)
$$

and $h_{i j}(0,0)=0$.

(H3) Time-varying delay function $\tau_{i j}(\cdot):[0,+\infty) \rightarrow$ $[0,+\infty)(i, j=1,2, \ldots, n)$ satisfies

$$
0 \leq \tau_{i j}(t) \leq \tau, \quad \dot{\tau}_{i j}(t) \leq \tau_{0} \leq 1,
$$

for $t \geq 0$, where $\tau$ and $\tau_{0}$ are constants.

It is well known, if the parameters or time-varying delay in neural networks is appropriately chosen, neural networks may lead to some phenomena such as instability, divergence, oscillation, chaos $[32,33]$.

In order to stabilize the origin of system (3)-(5), we introduce the following periodically intermittent controller:

$$
v_{i}(t, x)= \begin{cases}\sum_{j=1}^{n} k_{i j} u_{j}(t, x), & M T \leq t<M T+\delta, \\ 0, & M T+\delta \leq t<(M+1) T,\end{cases}
$$

where $M=0,1,2, \ldots$ and $k_{i j}$ is the control gains for $i, j=$ $1,2, \ldots, n, T$ denotes the control period, and $0<\delta<T$ is called the control width. 
Then, system (3) under the periodically intermittent controller (13) is described by the following equations:

$$
\begin{aligned}
& d u_{i}(t, x)=\left\{\sum_{k=1}^{m} \frac{\partial}{\partial x_{k}}\left(D_{i k}(l) \frac{\partial u_{i}(t, x)}{\partial x_{k}}\right)\right. \\
&-a_{i}(r(t)) u_{i}(t, x)+\sum_{j=1}^{n} b_{i j}(r(t)) f_{j}\left(u_{j}(t, x)\right) \\
&+\sum_{j=1}^{n} c_{i j}(r(t)) g_{j}\left(u_{j}\left(t-\tau_{i j}(t)\right), x\right) \\
&\left.+\sum_{j=1}^{n} k_{i j} u_{j}(t, x)\right\} d t \\
&+ \sum_{j=1}^{n} h_{i j}\left(u_{j}(t, x), u_{j}\left(t-\tau_{i j}(t), x\right)\right) d W_{j}(t) \\
&+\sum_{j=1}^{n} h_{i j}\left(u_{j}(t, x), u_{j}\left(t-\tau_{i j}(t), x\right)\right) d W_{j}(t) \\
& d u_{i}(t, x)=\left\{\begin{array}{l}
\sum_{k=1}^{m} \frac{\partial}{\partial x_{k}}\left(D_{i k}(l) \frac{\partial u_{i}(t, x)}{\partial x_{k}}\right) \\
\left.+\sum_{j=1}^{n} c_{i j}(r(t)) g_{j}\left(u_{j}\left(t-\tau_{i j}(t)\right), x\right)\right\} d t \\
\end{array}\right. \\
&-a_{i}(r(t)) u_{i}(t, x)+\sum_{j=1}^{n} b_{i j}(r(t)) f_{j}\left(u_{j}(t, x)\right)
\end{aligned}
$$

Lemma 3 (see [10]). Let $p \geq 2$ be a positive integer, let $\theta_{k}$ be a positive constant, let $\Omega_{0}$ be a cube $\left|x_{k}\right| \leq \theta_{k}$ for $k=1,2, \ldots, m$, and let $u(x)$ be a real-valued function belonging to $C^{1}\left(\Omega_{0}\right)$ which vanish on the boundary $\partial \Omega_{0}$; that is, $\left.u(x)\right|_{\partial \Omega_{0}}=0$; then

$$
\int_{\Omega_{0}}|u(x)|^{p} d x \leq \frac{p^{2} \theta_{k}^{2}}{4} \int_{\Omega_{0}}|u(x)|^{p-2}\left|\frac{\partial u}{\partial x_{k}}\right|^{2} d x .
$$

\section{Robust Exponential Stabilization}

In this section, we design suitable $T, \delta$, and $k_{i j}$ such that system (3)-(5) under the external controller (13) can realize robust exponential stability in $p$ th moment. For convenience, we give some denotations as follows:

$$
\begin{aligned}
\lambda_{i}=\min _{l \in \mathbb{S}} \mu_{l}\left\{\sum_{k=1}^{m} \frac{4(p-1) \underline{D}_{i k}}{p \theta_{k}^{2}}+p a_{i}^{*}\right. \\
-\sum_{j=1}^{n} \sum_{\ell=1}^{p-1}\left(\widetilde{b}_{i j}^{p \alpha_{\ell i j}} \tilde{L}_{j}^{p \beta_{\ell i j}}+\widetilde{c}_{i j}^{p \xi_{\ell i j}} \widetilde{N}_{j}^{p \zeta_{\ell i j}}\right) \\
-\frac{p-1}{2} \sum_{j=1 \ell=1}^{n} \sum_{\ell-2}^{p-2}\left(\sigma_{i j}^{p \epsilon_{i j}}+\sigma_{i j}^{p \omega_{i j}}\right) \\
-\sum_{j=1}^{n}\left(\widetilde{b}_{j i}^{p \alpha_{p j i}} \tilde{L}_{i}^{p \beta_{p j i}}\right. \\
+\widetilde{c}_{j i}^{p \xi_{p j i}} \widetilde{N}_{i}^{p \zeta_{p j i}} \\
\left.\left.+\frac{p-1}{2}\left(\sigma_{j i}^{p \epsilon_{(p-1) j i}}+\sigma_{j i}^{p \epsilon_{p j i}}\right)\right)\right\},
\end{aligned}
$$

$$
\begin{aligned}
& \kappa_{i}=\min _{l \in \mathbb{S}} \mu_{l}\left\{\sum_{k=1}^{m} \frac{4(p-1) \underline{D}_{i k}}{p \theta_{k}^{2}}+p a_{i}^{*}\right. \\
& -\sum_{j=1 \ell=1}^{n} \sum_{\ell=1}^{p-1}\left(\widetilde{b}_{i j}^{p \bar{\alpha}_{i j}} \tilde{L}_{j}^{p \bar{\beta}_{\ell i j}}+\widetilde{c}_{i j}^{p \bar{\xi}_{i j}} \widetilde{N}_{j}^{p \bar{\zeta}_{\ell i j}}\right) \\
& -\frac{p-1}{2} \sum_{j=1}^{n} \sum_{\ell=1}^{p-2}\left(\sigma_{i j}^{p \bar{\epsilon}_{\ell j}}+\sigma_{i j}^{p \bar{\omega}_{\ell i j}}\right) \\
& -\sum_{j=1}^{n}\left(\widetilde{b}_{j i}^{p \bar{\alpha}_{p j i}} \widetilde{L}_{i}^{p \bar{\beta}_{p j i}}+\widetilde{c}_{j i}^{p \bar{\xi}_{p j i}} \widetilde{N}_{i}^{p \bar{\zeta}_{p j i}}\right. \\
& \left.\left.+\frac{p-1}{2}\left(\bar{\sigma}_{j i}^{p \bar{\epsilon}_{(p-1) j i}}+\sigma_{j i}^{p \bar{\epsilon}_{p j i}}\right)\right)\right\},
\end{aligned}
$$

$$
\begin{gathered}
v_{i}=\max _{l \in \mathbb{S}} \mu_{l}\left[p k_{i i}+\sum_{\substack{j=1 \\
j \neq i}}^{n} \sum_{\ell=1}^{p-1}\left|k_{i j}\right|^{p \eta_{e i j}^{*}}\right. \\
\left.+\sum_{\substack{j=1 \\
j \neq i}}^{n}\left|k_{j i}\right|^{p \eta_{p j i}^{*}}\right], \\
\eta_{i}=\max _{l \in \mathbb{S}} \mu_{l} \sum_{j=1}^{n}\left[\frac{p-1}{2}\left(\sigma_{j i}^{\omega_{(p-1) j i}}+\sigma_{j i}^{\omega_{p j i}}\right)\right. \\
\left.+\widetilde{c}_{j i}^{p \xi_{p j i}} \widetilde{N}_{i}^{p \zeta_{p j i}}\right],
\end{gathered}
$$


where $a_{i}^{*}=\min _{l \in \mathbb{S}} \underline{a}_{i}(l), \tilde{b}_{i j}=\max _{l \in \mathbb{S}} \check{b}_{i j}(l), \check{b}_{i j}(l)=$ $\max \left\{\left|\underline{b}_{i j}(l)\right|,\left|\bar{b}_{i j}(l)\right|\right\}, \tilde{c}_{i j}=\max _{l \in \mathbb{S}} \check{c}_{i j}(l)$,

$$
\begin{aligned}
& \check{c}_{i j}(l)=\max \left\{\left|\underline{c}_{i j}(l)\right|,\left|\bar{c}_{i j}(l)\right|\right\}, \\
& \widetilde{L}_{j}=\max \left\{\left|L_{j}^{-}\right|,\left|L_{j}^{+}\right|\right\}, \quad \widetilde{N}_{j}=\max \left\{\left|N_{j}^{-}\right|,\left|N_{j}^{+}\right|\right\},
\end{aligned}
$$

$\mu_{l}>0$, and $\alpha_{\ell i j}, \beta_{\ell i j}, \xi_{\ell i j}, \zeta_{\ell i j}, \epsilon_{\ell i j}, w_{\ell i j}, \eta_{\ell i j}^{*}, \bar{\alpha}_{\ell i j}, \bar{\beta}_{\ell i j}, \bar{\xi}_{\ell i j}, \bar{\zeta}_{\ell i j}$, $\bar{\epsilon}_{\ell i j}$, and $\bar{w}_{\ell i j}$ are nonnegative constants, satisfying

$$
\begin{aligned}
\sum_{\ell=1}^{p} \alpha_{\ell i j} & =\sum_{\ell=1}^{p} \beta_{\ell i j}=\sum_{\ell=1}^{p} \xi_{\ell i j}=\sum_{\ell=1}^{p} \zeta_{\ell i j} \\
& =\sum_{\ell=1}^{p} \epsilon_{\ell i j}=\sum_{\ell=1}^{p} w_{\ell i j}=\sum_{\ell=1}^{p} \bar{\alpha}_{\ell i j}=\sum_{\ell=1}^{p} \bar{\beta}_{\ell i j} \\
& =\sum_{\ell=1}^{p} \bar{\xi}_{\ell i j}=\sum_{\ell=1}^{p} \bar{\zeta}_{\ell i j}=\sum_{\ell=1}^{p} \bar{\epsilon}_{\ell i j}=\sum_{\ell=1}^{p} \bar{w}_{\ell i j} \\
& =\sum_{\ell=1}^{p} \eta_{\ell i j}^{*}=1, \quad \underline{D}_{i k}=\min _{l \in \mathbb{S}} \underline{D}_{i k}(l) .
\end{aligned}
$$

In the following, we give an assumption:

(H4) $\lambda_{i}-v_{i}-\max _{l \in \mathbb{S}} \sum_{q=1}^{N} \gamma_{l q} \mu_{q}-\eta_{i} /\left(1-\tau_{0}\right)>0$ and there exists $\rho_{i}>0$ such that

$$
\kappa_{i}+\rho_{i}-\max _{l \in \mathbb{S}} \sum_{q=1}^{N} \gamma_{l q} \mu_{q}-\frac{\eta_{i}}{1-\tau_{0}}>0, \quad i=1,2, \ldots, n .
$$

We consider the function

$$
\begin{array}{r}
H_{i}\left(\check{\varepsilon}_{i}\right)=\lambda_{i}-v_{i}-\max _{l \in \mathbb{S}} \sum_{q=1}^{N} \gamma_{l q} \mu_{q}-\check{\varepsilon}_{i} \max _{l \in \mathbb{S}} \mu_{l}-\frac{\eta_{i} e^{\check{\varepsilon}_{i} \tau}}{1-\tau_{0}}, \\
i=1,2, \ldots, n .
\end{array}
$$

It is easy to see that

$$
\begin{aligned}
& H_{i}^{\prime}\left(\check{\varepsilon}_{i}\right)=-\max _{l \in \mathbb{S}} \mu_{l}-\frac{\tau \eta_{i} e^{\check{\varepsilon}_{i} \tau}}{1-\tau_{0}}<0, \\
& H_{i}(0)=\lambda_{i}-\nu_{i}-\max _{l \in \mathbb{S}} \sum_{q=1}^{N} \gamma_{l q} \mu_{q}-\frac{\eta_{i}}{1-\tau_{0}}>0 .
\end{aligned}
$$

On the other hand, $H_{i}\left(\check{\varepsilon}_{i}\right)$ is continuous on $[0,+\infty)$, and $F_{i}\left(\check{\varepsilon}_{i}\right) \rightarrow-\infty$ as $\check{\varepsilon}_{i} \rightarrow+\infty$. Then there exists a positive constant $\check{\varepsilon}_{i}^{*}$ such that $H_{i}\left(\check{\varepsilon}_{i}^{*}\right) \geq 0$ and $H_{i}\left(\check{\varepsilon}_{i}\right)>0$, for $\check{\varepsilon}_{i} \in$ $\left(0, \check{\varepsilon}_{i}^{*}\right)$.

$$
\begin{aligned}
& \text { Let } \check{\varepsilon}=\min _{1 \leq i \leq n}\left\{\check{\varepsilon}_{i}^{*}\right\} ; \text { then we have } \\
& H_{i}(\check{\varepsilon})=\lambda_{i}-v_{i}-\max _{l \in \mathbb{S}} \sum_{q=1}^{N} \gamma_{l q} \mu_{q}-\check{\varepsilon}_{l \in \mathbb{S}} \max _{l}-\frac{\eta_{i}}{1-\tau_{0}} e^{\check{\varepsilon} \tau} \geq 0 .
\end{aligned}
$$

In similar, there exists a positive constant $\widehat{\varepsilon}>0$, such that $F_{i}(\widehat{\varepsilon})=k_{i}+\rho_{i}-\max _{l \in \mathbb{S}} \sum_{q=1}^{N} \gamma_{l q} \mu_{q}-\widehat{\varepsilon} \max _{l \in \mathbb{S}} \mu_{l}-\frac{\eta_{i}}{1-\tau_{0}} e^{\widehat{\varepsilon} \tau} \geq 0$.

Let $\varepsilon=\min \{\check{\varepsilon}, \widehat{\varepsilon}\}$; we have

$$
H_{i}(\varepsilon)>0, \quad F_{i}(\varepsilon)>0, \quad i=1,2, \ldots, n .
$$

We give another assumption:

(H5) $\varepsilon-\rho(T-\delta) / \bar{\mu} T>0$, where $\rho=\max _{1 \leq i \leq n} \rho_{i}, \bar{\mu}=$ $\min _{l \in \mathbb{S}}\left\{\mu_{l}\right\}$.

Theorem 4. Under assumptions (H1)-(H5), the origin of system (3)-(5) under periodically intermittent controller (13) is robust exponentially stable in pth moment.

Proof. Let us define the average Lyapunov-Krasovskii functional (see [4]) $V_{1}: C\left([0,+\infty) \times \Omega_{0}, \mathbb{R}^{n}\right) \times \mathbb{S} \times \mathbb{R}_{+} \rightarrow \mathbb{R}_{+}$ by

$$
V_{1}(u(t, x), r(t), t)=\int_{\Omega_{0}} V(u(t, x), r(t), t) d x
$$

with

$$
\begin{aligned}
& V(u(t, x), r(t), t) \\
& =\mu_{r(t)} \sum_{i=1}^{n} e^{\varepsilon t}\left|u_{i}(t, x)\right|^{p}+\frac{e^{\varepsilon \tau}}{1-\tau_{0}} \sum_{i=1}^{n} \eta_{i} \int_{t-\tau_{i j}(t)}^{t} e^{\varepsilon s}\left|u_{i}(s, x)\right|^{p} d s,
\end{aligned}
$$

where $\mu_{r(t)}>0$.

By the generalized Itô formula (see [31]), we have

$$
\begin{aligned}
& \mathbb{E} V_{1}(u(t, x), r(t), t) \\
& =\mathbb{E} V_{1}(\phi, r(0), 0)+\mathbb{E} \int_{0}^{t} \int_{\Omega_{0}} \mathscr{L} V(u(s, x), r(s), s) d x d s .
\end{aligned}
$$

By Lemma 3.1 in [22] and $r(t)=l$, we get that for $(t, x) \in$ $[M T, M T+\delta) \times \Omega_{0}$

$$
\begin{aligned}
& \mathscr{L} V(u(t, x), l, t) \\
& =\varepsilon \mu_{l} \sum_{i=1}^{n} e^{\varepsilon t}\left|u_{i}(t, x)\right|^{p}+p \mu_{l} e^{\varepsilon t} \sum_{i=1}^{n}\left|u_{i}(t, x)\right|^{p-1} \\
& \times\left\{\sum_{k=1}^{m} \frac{\partial}{\partial x_{k}}\left(D_{i k}(l) \frac{\partial u_{i}(t, x)}{\partial x_{k}}\right)-a_{i}(l) u_{i}(t, x)\right. \\
& +\sum_{j=1}^{n} b_{i j}(l) f_{j}\left(u_{j}(t, x)\right) \\
& +\sum_{j=1}^{n} c_{i j}(l) g_{j}\left(u_{j}\left(t-\tau_{i j}(t), x\right)\right) \\
& \left.+\sum_{j=1}^{n} k_{i j} u_{j}(t, x)\right\}
\end{aligned}
$$




$$
\begin{aligned}
& +\mu_{l} e^{\varepsilon t} \frac{p(p-1)}{2} \sum_{i=1}^{n}\left|u_{i}(t, x)\right|^{p-2} \\
& \times \sum_{j=1}^{n} h_{i j}^{2}\left(u_{j}(t, x), u_{j}\left(t-\tau_{i j}(t), x\right)\right) \\
& +\sum_{q=1}^{N} \gamma_{l q} \mu_{q} e^{\varepsilon t} \sum_{i=1}^{n}\left|u_{i}(t, x)\right|^{p} \\
& +\frac{e^{\varepsilon \tau}}{1-\tau_{0}} \sum_{i=1}^{n} \eta_{i} e^{\varepsilon t}\left|u_{i}(t, x)\right|^{p} \\
& -\frac{e^{\varepsilon \tau}}{1-\tau_{0}} \sum_{i=1}^{n} \eta_{i} e^{\varepsilon\left(t-\tau_{i j}(t)\right)} \\
& \times\left|u_{i}\left(t-\tau_{i j}(x), x\right)\right|^{p}\left(1-\dot{\tau}_{i j}(t)\right) \\
& +\sum_{q=1}^{N} \gamma_{l q} \frac{e^{\varepsilon \tau}}{1-\tau_{0}} \sum_{i=1}^{n} \eta_{i} \int_{t-\tau_{i j}(t)}^{t} e^{\varepsilon s}\left|u_{i}(s, x)\right|^{p} d s .
\end{aligned}
$$

By the fundamental inequality $|a+b| \leq|a|+|b|$, we have

$$
\begin{aligned}
& \mathscr{L} V(u(t, x),l, t) \\
& \leq \sum_{i=1}^{n} e^{\varepsilon t}\left\{\varepsilon \mu_{l}\left|u_{i}(t, x)\right|^{p}+p \mu_{l}\left|u_{i}(t, x)\right|^{p-1}\right. \\
& \times\left(\sum_{k=1}^{m} \frac{\partial}{\partial x_{k}}\left(D_{i k}(l)\right) \frac{\partial u_{i}(t, x)}{\partial x_{k}}\right) \\
&+\left(k_{i i}-a_{i}(l)\right) p \mu_{l}\left|u_{i}(t, x)\right|^{p} \\
&+p \mu_{l}\left|u_{i}(t, x)\right|^{p-1}\left(\sum_{j=1}^{n}\left|b_{i j}(l)\right|\left|f_{j}\left(u_{j}(t, x)\right)\right|\right) \\
&+p \mu_{l}\left|u_{i}(t, x)\right|^{p-1} \\
& \times\left(\sum_{j=1}^{n}\left|c_{i j}(l)\right|\left|g_{j}\left(\mu_{j}\left(t-\tau_{i j}(t)\right), x\right)\right|\right) \\
&+\sum_{q=1}^{n} p \mu_{l}\left|u_{i}(t, x)\right|^{p-1}\left|k_{i j}\right|\left|\mu_{j}(t, x)\right| \\
&+\sum_{j=1}^{n} \sigma_{i j}\left(\left|u_{j}(t, x)\right|^{2}+\left|u_{j}\left(t-\tau_{i j}(t), x\right)\right|^{2}\right) \\
&+\left.\mu_{l} \frac{p(p-1)}{2}\left|u_{i}(t, x)\right|^{p-2}(t, x)\right|^{p} \\
& \\
& \\
& \\
& \\
&
\end{aligned}
$$

$$
\begin{aligned}
& +\frac{e^{\varepsilon \tau}}{1-\tau_{0}} \eta_{i}\left|u_{i}(t, x)\right|^{p} \\
& \left.-\eta_{i}\left|u_{i}\left(t-\tau_{i j}(t), x\right)\right|^{p}\right\},
\end{aligned}
$$

where we use $\sum_{q=1}^{N} \gamma_{l q}=0$.

By using the fundamental inequality $a_{1}^{p}+a_{2}^{p}+\cdots+a_{p}^{p} \geq$ $p a_{1} a_{2}, \ldots, a_{p}\left(a_{i} \geq 0, i=1,2, \ldots, p\right)$, we have

$$
\begin{aligned}
& p \mu_{l}\left|u_{i}(t, x)\right|^{p-1}\left(\sum_{j=1}^{n}\left|b_{i j}(l)\right|\left|f_{j}\left(u_{j}(t, x)\right)\right|\right) \\
& \leq \mu_{l} \sum_{j=1}^{n} p\left|u_{i}(t, x)\right|^{p-1} \widetilde{b}_{i j} \widetilde{L}_{j}\left|u_{j}(t, x)\right| \\
& =\mu_{l} \sum_{j=1}^{n} p\left[\prod_{\ell=1}^{p-1} \widetilde{b}_{i j}^{\alpha_{\ell j}} \widetilde{L}_{j}^{\beta_{\ell i j}}\left|u_{i}(t, x)\right|\right]
\end{aligned}
$$$$
\times\left(\widetilde{b}_{i j}^{\alpha_{p i j}} \widetilde{L}_{j}^{\beta_{p i j}}\left|u_{j}(t, x)\right|\right)
$$$$
\leq \mu_{l} \sum_{j=1}^{n} \sum_{\ell=1}^{p-1} \widetilde{b}_{i j}^{p \alpha_{\ell i j}} \widetilde{L}_{j}^{p \beta_{\ell i j}}\left|u_{i}(t, x)\right|^{p}
$$$$
+\mu_{l} \sum_{j=1}^{n} \widetilde{b}_{i j}^{p \alpha_{p i j}} \widetilde{L}_{j}^{p \beta_{p i j}}\left|u_{j}(t, x)\right|^{p},
$$

$p \mu_{l}\left|u_{i}(t, x)\right|^{p-1}$

$$
\begin{aligned}
& \times\left(\sum_{j=1}^{n}\left|c_{i j}(l)\right|\left|g_{j}\left(u_{j}\left(t-\tau_{i j}(t), x\right)\right)\right|\right) \\
& \leq p \mu_{l} \sum_{j=1}^{n}\left|u_{i}(t, x)\right|^{p-1} \widetilde{c}_{i j} \widetilde{N}_{j}\left|u_{j}\left(t-\tau_{i j}(t), x\right)\right| \\
& =\mu_{l} \sum_{j=1}^{n} p\left[\prod_{\ell=1}^{p-1} \widetilde{c}_{i j}^{\xi_{\ell i j}} \widetilde{N}_{j}^{\zeta_{i j}}\left|u_{j}(t, x)\right|\right] \\
& \times\left(\widetilde{c}_{i j}^{\xi_{p i j}} \widetilde{N}_{j}^{\zeta_{p i j}}\left|u_{j}\left(t-\tau_{i j}(t), x\right)\right|\right) \\
& \leq \mu_{l} \sum_{j=1}^{n} \sum_{\ell=1}^{p-1} \widetilde{c}_{i j}^{p \xi_{\ell i j}} \widetilde{N}_{j}^{p \zeta_{\ell i j}}\left|u_{j}(t, x)\right|^{p} \\
& +\mu_{l} \sum_{j=1}^{n} \widetilde{c}_{i j}^{p \xi_{p i j}} \widetilde{N}_{j}^{p \zeta_{p i j}}\left|u_{j}\left(t-\tau_{i j}(t), x\right)\right|^{p} .
\end{aligned}
$$


Similarly, we have

$$
\begin{aligned}
& \mu_{l} \sum_{\substack{j=1 \\
j \neq i}}^{n} p\left|u_{i}(t, x)\right|^{p-1}\left|k_{i j}\right|\left|u_{j}(t, x)\right| \\
& =\mu_{l} \sum_{\substack{j=1 \\
j \neq i}}^{n} p\left[\prod_{\ell=1}^{p-1}\left|k_{i j}\right|^{\eta_{\ell i j}^{*}}\left|u_{i}(t, x)\right|\right] \\
& \times\left(\left|k_{i j}\right|^{\eta_{p i j}^{*}}\left|u_{j}(t, x)\right|\right) \\
& \leq \mu_{l} \sum_{\substack{j=1 \\
j \neq i}}^{n} \sum_{\ell=1}^{p-1}\left|k_{i j}\right|^{p \eta_{\ell i j}^{*}}\left|u_{i}(t, x)\right|^{p} \\
& +\mu_{l} \sum_{\substack{j=1 \\
j \neq i}}^{n}\left|k_{i j}\right|^{p \eta_{p i j}^{*}}\left|u_{j}(t, x)\right|^{p} \\
& \mu_{l} \frac{p(p-1)}{2}\left|u_{i}(t, x)\right|^{p-2} \\
& \times \sum_{j=1}^{n} \sigma_{i j}\left|u_{j}(t, x)\right|^{2} \\
& =\mu_{l} \frac{p-1}{2} \sum_{j=1}^{n} p\left[\prod_{\ell=1}^{p-2}\left|\sigma_{i j}\right|^{\epsilon_{\ell i j}}\left|u_{i}(t, x)\right|\right] \\
& \times\left(\left|\sigma_{i j}\right|^{\epsilon_{(p-1) i j}}\left|u_{j}(t, x)\right|\right)\left(\left|\sigma_{i j}\right|^{\epsilon_{p i j}}\left|u_{j}(t, x)\right|\right) \\
& \leq \mu_{l} \frac{p-1}{2} \sum_{j=1}^{n} \sum_{\ell=1}^{p-2}\left|\sigma_{i j}\right|^{p \epsilon_{\ell i j}}\left|u_{i}(t, x)\right|^{p} \\
& +\mu_{l} \frac{p-1}{2} \sum_{j=1}^{n}\left(\left|\sigma_{i j}\right|^{p \epsilon_{(p-1) i j}}+\left|\sigma_{i j}\right|^{p \epsilon_{p i j}}\right) \\
& \times\left|u_{j}(t, x)\right|^{p} .
\end{aligned}
$$

Further, we also have

$$
\begin{aligned}
& \mu_{l} \frac{p(p-1)}{2}\left|u_{i}(t, x)\right|^{p-2} \\
& \quad \times \sum_{j=1}^{n} \sigma_{i j}\left|u_{j}\left(t-\tau_{i j}(t), x\right)\right|^{2} \\
& =\mu_{l} \frac{p-1}{2} \sum_{j=1}^{n} p\left[\prod_{\ell=1}^{p-2} \sigma_{i j}^{\omega_{\ell i j}}\left|u_{i}(t, x)\right|\right] \\
& \quad \times\left(\sigma_{i j}^{\omega_{(p-1) i j}}\left|u_{j}\left(t-\tau_{i j}(t), x\right)\right|\right) \\
& \quad \times\left(\sigma_{i j}^{\omega_{p i j}}\left|u_{j}\left(t-\tau_{i j}(t), x\right)\right|\right)
\end{aligned}
$$

$$
\begin{aligned}
& \leq \mu_{l} \frac{p-1}{2} \sum_{j=1}^{n} \sum_{\ell=1}^{p-2}\left|\sigma_{i j}\right|^{p \omega_{\ell i j}}\left|u_{i}(t, x)\right|^{p} \\
& \quad+\mu_{l} \frac{p-1}{2} \sum_{j=1}^{n}\left(\sigma_{i j}^{\omega_{(p-1) i j}}+\sigma_{i j}^{\omega_{p i j}}\right) \\
& \quad \times\left|u_{j}\left(t-\tau_{i j}(t), x\right)\right|^{p} .
\end{aligned}
$$

Substituting (33)-(35) into (32), we obtain

$$
\mathscr{L} V(u(t, x), l, t)
$$$$
\leq \sum_{i=1}^{n} e^{\varepsilon t}\left\{\left[\mu_{l}+p \mu_{l} k_{i i}-p \mu_{l} a_{i}^{*}\right.\right.
$$$$
+\sum_{q=1}^{N} \gamma_{l q} \mu_{q}+\frac{e^{\varepsilon \tau} \eta_{i}}{1-\tau_{0}}
$$$$
+\mu_{l} \sum_{j=1}^{n} \sum_{\ell=1}^{p-1}\left(\widetilde{b}_{i j}^{p \alpha_{\ell i j}} \widetilde{L}_{j}^{p \beta_{\ell i j}}+\widetilde{c}_{i j}^{p \xi_{\ell i j}} \widetilde{N}_{j}^{p \zeta_{\ell i j}}\right)
$$$$
+\mu_{l} \sum_{\substack{j=1 \\ j \neq i}}^{n} \sum_{\ell=1}^{p-1}\left|k_{i j}\right|^{p \eta_{\ell i j}^{*}}
$$$$
\left.+\mu_{l} \frac{p-1}{2} \sum_{j=1}^{n} \sum_{\ell=1}^{p-2}\left(\sigma_{i j}^{p \epsilon_{\ell i j}}+\sigma_{i j}^{p \omega_{\ell i j}}\right)\right]
$$$$
\times\left|u_{i}(t, x)\right|^{p}
$$$$
+\mu_{l} \sum_{j=1}^{n}\left[\widetilde{b}_{i j}^{p \alpha_{p i j}} \widetilde{L}_{j}^{p \beta_{p i j}}+\widetilde{c}_{i j}^{p \xi_{p i j}} \widetilde{N}_{j}^{p \zeta_{p i j}}\right.
$$$$
\left.+\frac{p-1}{2}\left(\sigma_{i j}^{p \epsilon_{(p-1) i j}}+\sigma_{i j}^{p \epsilon_{p i j}}\right)\right]
$$

$\times\left|u_{i}(t, x)\right|^{p}+\mu_{l} \sum_{\substack{j=1 \\ j \neq i}}^{n}\left|k_{i j}\right|^{p \eta_{p i j}^{*}}\left|u_{j}(t, x)\right|^{p}$

$$
\begin{aligned}
& +\mu_{l} \frac{p-1}{2} \sum_{j=1}^{n}\left(\sigma_{i j}^{\omega_{(p-1) i j}}+\sigma_{i j}^{\omega_{p i j}}\right) \\
& \times\left|u_{j}\left(t-\tau_{i j}(t), x\right)\right|^{p} \\
& +\mu_{l} \sum_{j=1}^{n} \widetilde{c}_{i j}^{p \xi_{p i j}} \widetilde{N}_{j}^{p \zeta_{p i j}}\left|u_{j}\left(t-\tau_{i j}(t), x\right)\right|^{p} \\
& \left.-\eta_{i}\left|u_{i}\left(t-\tau_{i j}(t), x\right)\right|^{p}\right\}
\end{aligned}
$$

$+\sum_{i=1}^{n} e^{\varepsilon t} p \mu_{l}\left|u_{i}(t, x)\right|^{p-1}$ 


$$
\begin{aligned}
& \times\left(\sum_{k=1}^{m} \frac{\partial}{\partial x_{k}}\left(D_{i k}(l) \frac{\partial u_{i}(t, x)}{\partial x_{k}}\right)\right) \\
\leq & \sum_{i=1}^{n} e^{\varepsilon t}\left\{\varepsilon \max _{l \in \mathbb{S}} \mu_{l}+v_{i}-\bar{\lambda}_{i}+\max _{l \in \mathbb{S}} \sum_{q=1}^{N} \gamma_{l q} \mu_{q}+\frac{e^{\varepsilon \tau} \eta_{i}}{1-\tau_{0}}\right\} \\
& \times\left|u_{i}(t, x)\right|^{p}+\sum_{i=1}^{n} e^{\varepsilon t} p \mu_{l}\left|u_{i}(t, x)\right|^{p-1} \\
& \times\left(\sum_{k=1}^{m} \frac{\partial}{\partial x_{k}}\left(D_{i k}(l) \frac{\partial u_{i}(t, x)}{\partial x_{k}}\right)\right)
\end{aligned}
$$

where

$$
\begin{gathered}
\bar{\lambda}_{i}=\min _{l \in \mathbb{S}} \mu_{l}\left\{p a_{i}^{*}-\sum_{j=1}^{n} \sum_{\ell=1}^{p-1}\left(\widetilde{b}_{i j}^{p \alpha_{\ell i j}} \widetilde{L}_{j}^{p \beta_{\ell i j}}+\widetilde{c}_{i j}^{p \xi_{\ell i j}} \widetilde{N}_{j}^{p \zeta_{\ell i j}}\right)\right. \\
-\frac{p-1}{2} \sum_{j=1}^{n} \sum_{\ell=1}^{p-2}\left(\sigma_{i j}^{p \epsilon_{\ell i j}}+\sigma_{i j}^{p \omega_{\ell i j}}\right) \\
-\sum_{j=1}^{n}\left(\widetilde{b}_{j i}^{p \alpha_{p j i}} \widetilde{L}_{i}^{p \beta_{p j i}}+\widetilde{c}_{j i}^{p \xi_{p j i}} \widetilde{N}_{i}^{p \zeta_{p j i}}\right. \\
\left.\left.+\frac{p-1}{2}\left(\sigma_{j i}^{p \epsilon_{(p-1) j i}}+\sigma_{j i}^{p \epsilon_{p j i}}\right)\right)\right\} .
\end{gathered}
$$

Substituting (36) into (30), we obtain

$$
\begin{aligned}
& \mathbb{E} V_{1}(u(t, x), r(t), t) \\
& \leq \mathbb{E} V_{1}(\phi, r(0), 0) \\
& -\mathbb{E} \int_{0}^{t} \int_{\Omega_{0}} \sum_{i=1}^{n} e^{\varepsilon s}\left[\bar{\lambda}_{i}-\varepsilon \max _{l \in \mathbb{S}} \mu_{l}-\nu_{i}\right. \\
& \left.-\max _{l \in \mathbb{S}} \sum_{q=1}^{N} \gamma_{l q} \mu_{q}-\frac{e^{\varepsilon \tau} \eta_{i}}{1-\tau_{0}}\right]
\end{aligned}
$$

$\times\left|u_{i}(s, x)\right|^{p} d x d s$

$$
\begin{aligned}
& +\mathbb{E} \int_{0}^{t} \sum_{i=1}^{n} e^{\varepsilon s} \int_{\Omega_{0}} p \mu_{r(s)}\left|u_{i}(s, x)\right|^{p-1} \\
& \times\left(\sum_{k=1}^{m} \frac{\partial}{\partial x_{k}}\left(D_{i k}(r(s)) \frac{\partial u_{i}(s, x)}{\partial x_{k}}\right)\right) d x d s .
\end{aligned}
$$

By Lemma 3 and the boundary condition (4), we have

$$
\begin{aligned}
& \int_{\Omega_{0}} p\left|u_{i}(s, x)\right|^{p-1} \\
& \quad \times\left(\sum_{k=1}^{m} \frac{\partial}{\partial x_{k}}\left(D_{i k}(r(s)) \frac{\partial u_{i}(s, x)}{\partial x_{k}}\right)\right) d x
\end{aligned}
$$

$$
\begin{aligned}
& \leq-\sum_{k=1}^{m} \frac{4(p-1) D_{i k}(r(s))}{p \theta_{k}^{2}} \int_{\Omega_{0}}\left|u_{i}(s, x)\right|^{p} d x \\
& \leq-\sum_{k=1}^{m} \frac{4(p-1) \underline{D}_{i k}}{p \theta_{k}^{2}} \int_{\Omega_{0}}\left|u_{i}(s, x)\right|^{p} d x .
\end{aligned}
$$

Substituting these into (38), we get

$$
\begin{aligned}
& \mathbb{E} V_{1}(u(t, x), r(t), t) \\
& \leq \mathbb{E} V_{1}(\phi, r(0), 0) \\
& -\mathbb{E} \int_{0}^{t} \int_{\Omega_{0}} \sum_{i=1}^{n} e^{\varepsilon s}\left[\bar{\lambda}_{i}+\min _{l \in \mathbb{S}} \mu_{l} \sum_{k=1}^{m} \frac{4(p-1) \underline{D_{i k}}}{p \theta_{k}^{2}}\right. \\
& -\varepsilon \max _{l \in \mathbb{S}} \mu_{l}-\nu_{i}-\max _{l \in \mathbb{S}} \sum_{q=1}^{N} \gamma_{l q} \mu_{q} \\
& \left.\leq-\frac{e^{\varepsilon \tau} \eta_{i}}{1-\tau_{0}}\right]\left|u_{i}(s, x)\right|^{p} d x d s \\
& -\mathbb{E} \int_{0}^{t} \int_{\Omega_{0}} \sum_{i=1}^{n} e^{\varepsilon s}\left[\lambda_{i}-\varepsilon \max _{l \in \mathbb{S}} \mu_{l}-\nu_{i}\right. \\
& \left.-\max _{l \in \mathbb{S}} \sum_{q=1}^{N} \gamma_{l q} \mu_{q}-\frac{e^{\varepsilon \tau} \eta_{i}}{1-\tau_{0}}\right]
\end{aligned}
$$

$\times\left|u_{i}(s, x)\right|^{p} d x d s$

$$
\leq \mathbb{E} V_{1}(\phi, r(0), 0)(t, x) \in[M T, M T+\delta) \times \Omega_{0} .
$$

Similarly, for $(t, x) \in[M T+\delta,(M+1) T) \times \Omega_{0}$, we can obtain

$$
\begin{aligned}
& \mathbb{E} V_{1}(u(t, x), r(t), t) \\
& \leq \mathbb{E} V_{1}(u(M T+\delta, x), r(M T+\delta), M T+\delta) \\
& -\mathbb{E} \int_{0}^{t} \int_{\Omega_{0}} \sum_{i=1}^{n} e^{\varepsilon s}\left[\kappa_{i}+\rho_{i}-\varepsilon \max _{l \in \mathbb{S}} \mu_{l}\right. \\
& \left.\quad \times \max _{l \in \mathbb{S}} \sum_{q=1}^{N} \gamma_{l q} \mu_{q}-\frac{e^{\varepsilon \tau} \eta_{i}}{1-\tau_{0}}\right] \\
& +\mathbb{E} \int_{0}^{t} \int_{\Omega_{0}} \sum_{i=1}^{n} e^{\varepsilon s} \rho_{i}\left|u_{i}(s, x)\right|^{p} d x d s \\
& \leq \mathbb{E} V_{1}(u(M T+\delta, x), r(M T+\delta), M T+\delta) \\
& +\frac{\rho}{\bar{\mu}} \mathbb{E} \int_{0}^{t} V_{1}(u(s, x), r(s), s) d s,
\end{aligned}
$$

where $\rho=\max _{1 \leq i \leq n} \rho_{i}, \bar{\mu}=\min _{l \in \mathbb{S}} \mu_{l}$. 


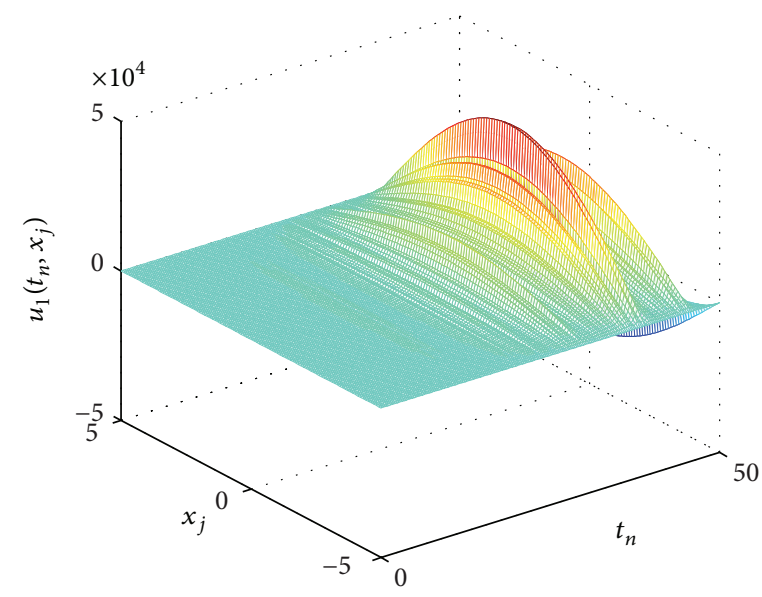

(a)

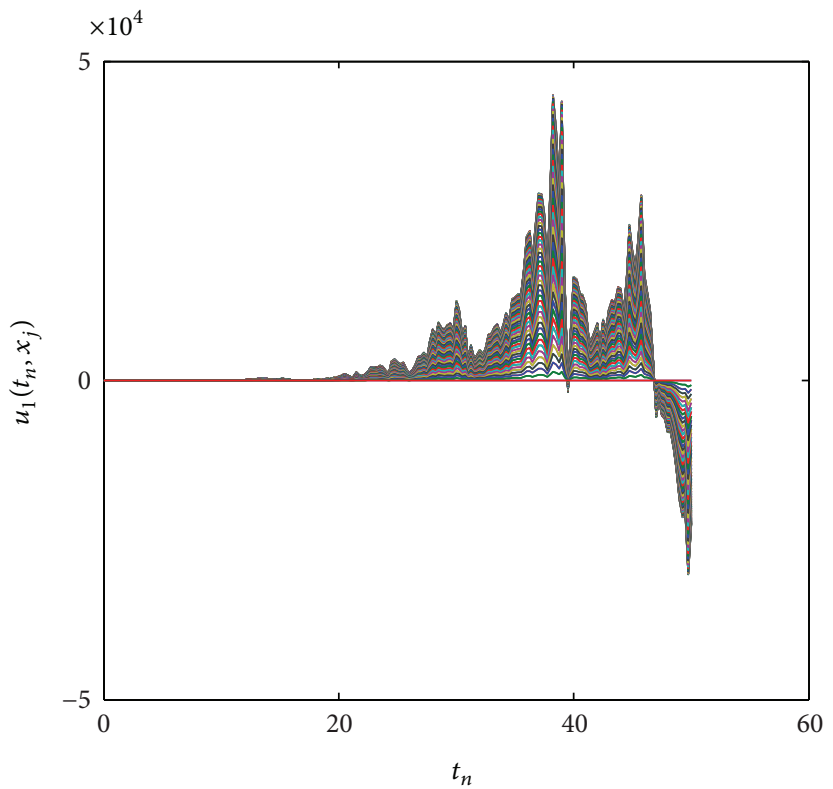

(c)

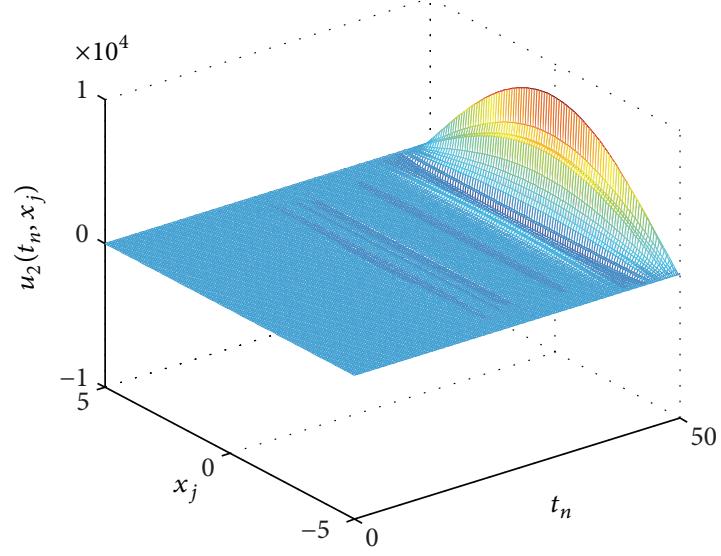

(b)

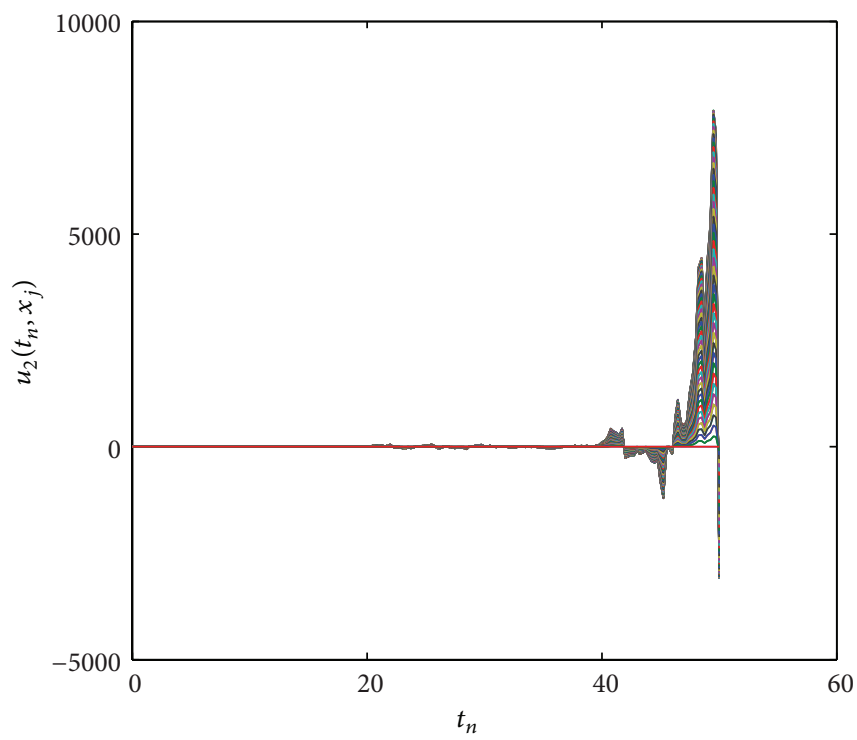

(d)

FIGURE 1: Surface curves and state trajectories for system (54) in model 1.

By the Gronwall inequality, we have

$$
\begin{aligned}
& \mathbb{E} V_{1}(u(t, x), r(t), t) \\
& \leq \mathbb{E} V_{1}(u(M T+\delta, x), \\
& \quad r(M T+\delta), M T+\delta) e^{(\rho / \bar{\mu})(t-M T-\delta)} .
\end{aligned}
$$

Combining (40) and (42), we summarize that,

(I) for $(t, x) \in[0, \delta) \times \Omega_{0}$, from (40), we have

$$
\mathbb{E} V_{1}(u(t, x), r(t), t) \leq \mathbb{E} V_{1}(u(0, x), r(0), 0) .
$$

(II) For $(t, x) \in[\delta, T) \times \Omega_{0}$, from (42), we get

$$
\begin{aligned}
& \mathbb{E} V_{1}(u(t, x), r(t), t) \\
& \quad \leq \mathbb{E} V_{1}(u(0, x), r(0), 0) e^{(\rho / \bar{\mu})(t-\delta)} .
\end{aligned}
$$

(III) For $(t, x) \in[T, T+\delta) \times \Omega_{0}$, from (40), we have

$$
\begin{aligned}
& \mathbb{E} V_{1}(u(t, x), r(t), t) \\
& \leq \mathbb{E} V_{1}(u(T, x), r(T), T) \\
& \leq \mathbb{E} V_{1}(u(0, x), r(0), 0) e^{(\rho / \bar{\mu})(T-\delta)} .
\end{aligned}
$$

(IV) For $(t, x) \in[T+\delta, 2 T) \times \Omega_{0}$, from (42), we have

$$
\begin{aligned}
& \mathbb{E} V_{1}(u(t, x), r(t), t) \\
& \quad \leq \mathbb{E} V_{1}(u(T+\delta, x), r(T+\delta), T+\delta) e^{(\rho / \bar{\mu})(t-T-\delta)} \\
& \leq \mathbb{E} V_{1}(u(0, x), r(0), 0) e^{(\rho / \bar{\mu})(t-2 \delta)} .
\end{aligned}
$$




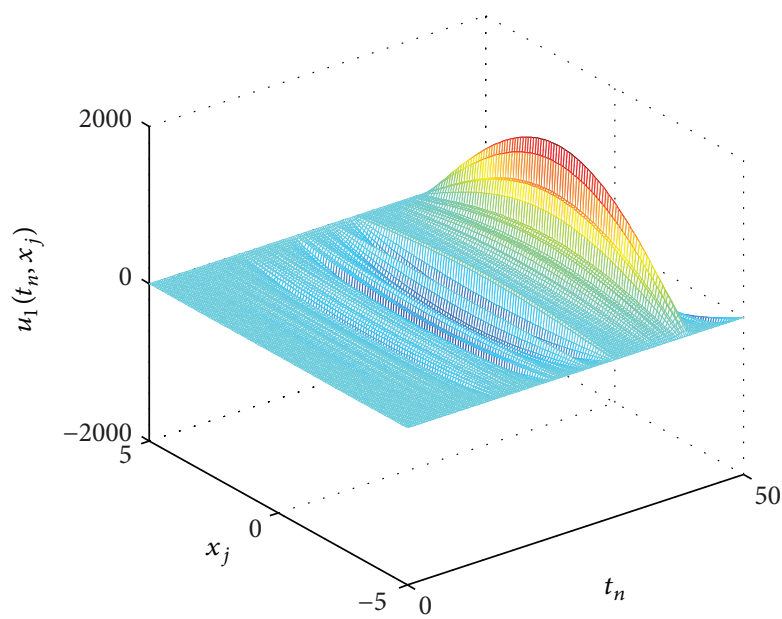

(a)

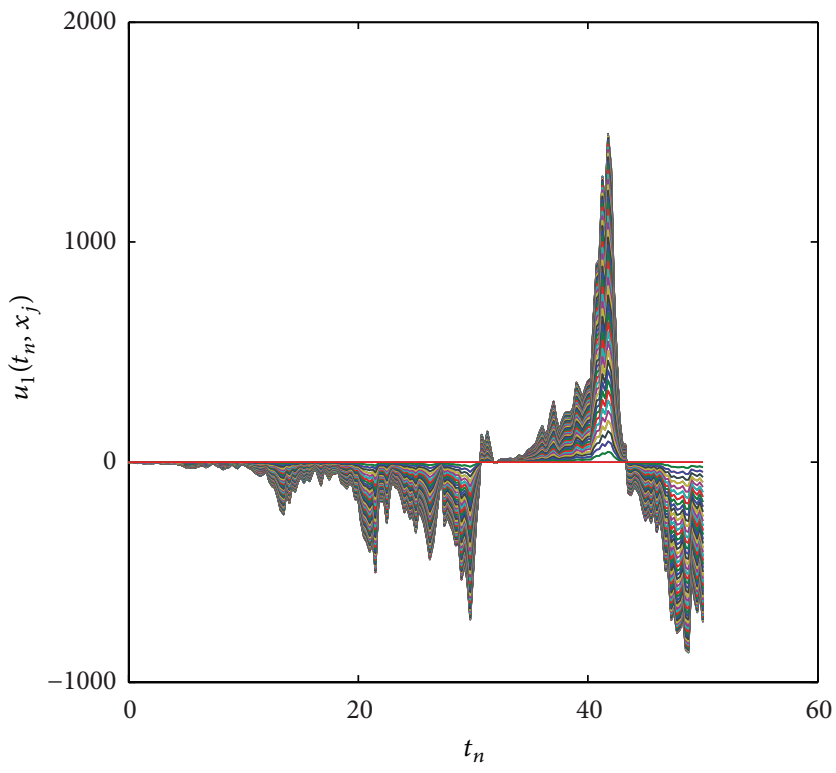

(c)

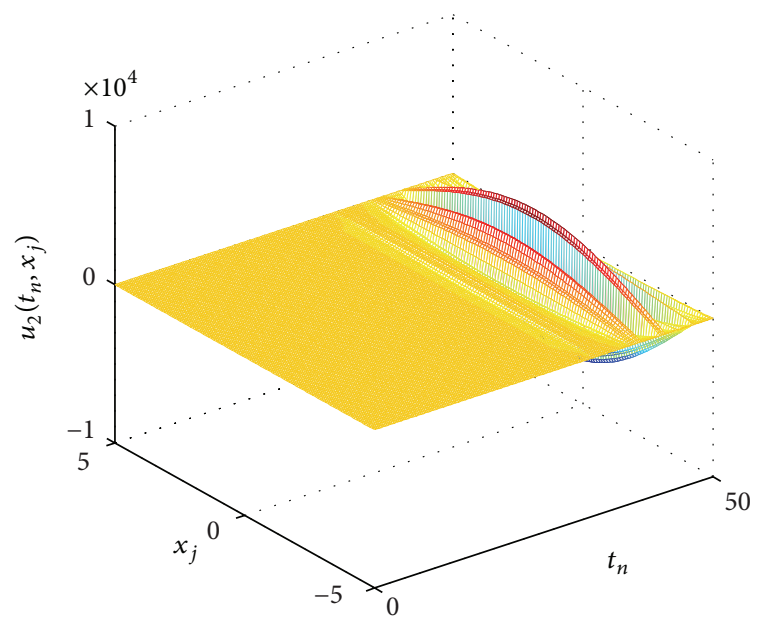

(b)

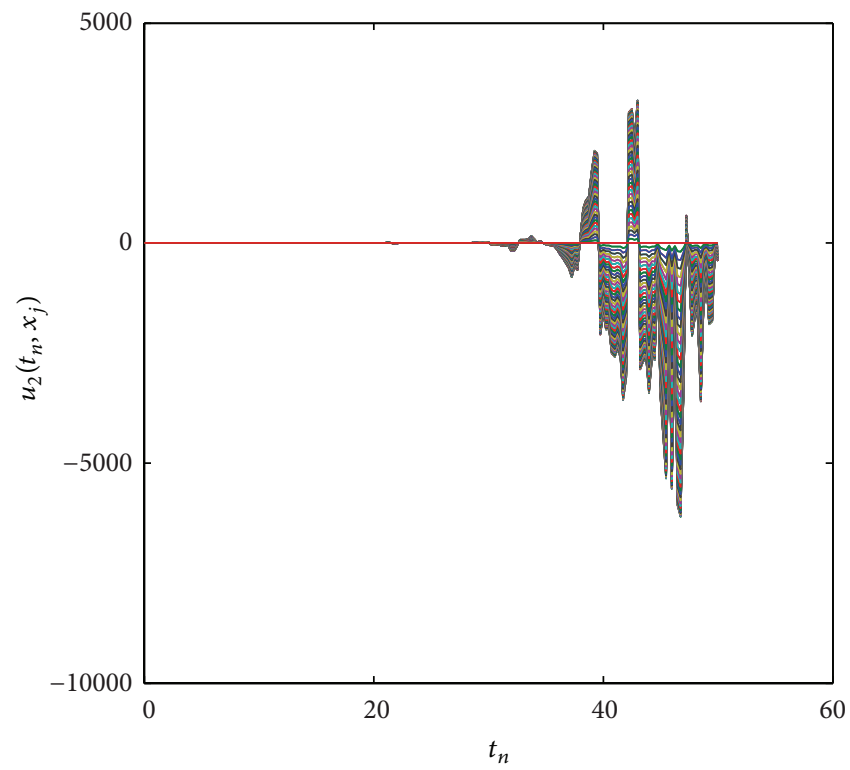

(d)

Figure 2: Surface curves and state trajectories for system (54) in model 2.

Repeating the above procedure, we obtain that, for $(t, x) \in$ $[M T, M T+\delta), M \leq(t / T)$,

$$
\begin{aligned}
& \mathbb{E} V_{1}(u(t, x), r(t), t) \\
& \quad \leq \mathbb{E} V_{1}(u(M T, x), r(M T), M T) \\
& \quad \leq \mathbb{E} V_{1}(u(0, x), r(0), 0) e^{(\rho(T-\delta) / \bar{\mu} T) t .}
\end{aligned}
$$

Moreover, for $(t, x) \in[M T+\delta,(M+1) T) \times \Omega_{0},(t / T)<$ $M+1$,

$\mathbb{E} V_{1}(u(t, x), r(t), t)$

$\leq \mathbb{E} V_{1}(\mu(M T+\delta, x), r(M T+\delta), M T+\delta) e^{(\rho / \bar{\mu})(t-M T-\delta)}$

$\leq \mathbb{E} V_{1}(u(0, x), r(0), 0) e^{(\rho / \bar{\mu})((T-\delta) / T) t}$.
Hence, for any $(t, x) \in[0,+\infty) \times \Omega_{0}$, we always have

$$
\mathbb{E} V_{1}(u(t, x), r(t), t) \leq \mathbb{E} V_{1}(u(0, x), r(0), 0) e^{(\rho / \bar{\mu})((T-\delta) / T) t}
$$

By (28) and (49), we have

$$
\begin{aligned}
& e^{\varepsilon t} \bar{\mu} \mathbb{E} \int_{\Omega_{0}} \sum_{i=1}^{n}\left|u_{i}(t, x)\right|^{p} d x \\
& \leq \mathbb{E} V_{1}(u(t, x), r(t), t) \\
& \leq \mathbb{E} V_{1}(u(0, x), r(0), 0) e^{(\rho / \bar{\mu})((T-\delta) / T) t} .
\end{aligned}
$$




$$
\begin{aligned}
& \text { Note that } \\
& \mathbb{E} V_{1}(u(0, x), r(0), 0) \\
& =\mathbb{E} \int_{\Omega_{0}} \mu_{l} \sum_{i=1}^{n}\left|u_{i}(0, x)\right|^{p} d x \\
& \quad+\frac{e^{\varepsilon \tau}}{1-\tau_{0}} \mathbb{E} \int_{\Omega_{0}} \sum_{i=1}^{n} \eta_{i} \int_{-\tau_{i j}(0)}^{0} e^{\varepsilon s}\left|u_{i}(s, x)\right|^{p} d s d x \\
& \leq \max _{l \in \mathbb{S}} \mu_{l} \mathbb{E} \int_{\Omega_{0}} \sum_{i=1}^{n}\left|\phi_{i}(0, x)\right|^{p} d x \\
& \quad+\sup _{-\tau \leq s \leq 0}\left[\left(\max _{1 \leq i \leq n} \eta_{i}\right) \frac{\tau e^{\varepsilon \tau}}{1-\tau_{0}} \mathbb{E} \int_{\Omega_{0}} \sum_{i=1}^{n}\left|\phi_{i}(s, x)\right|^{p} d x\right] \\
& =M_{0} .
\end{aligned}
$$

Under assumption (H5), the assertion of Theorem 4 follows from (50) and (51).

Corollary 5. Under assumptions (H1)-(H3), the origin of system (3)-(5) under periodically intermittent control (13) is robust exponentially stable in pth moment if the following conditions hold:

$$
\begin{aligned}
& \text { (I) } v_{i}<0, \lambda_{i}-v_{i}-\max _{l \in \mathbb{S}} \sum_{q=1}^{N} \gamma_{l q} \mu_{q}-\left(\eta_{i} /\left(1-\tau_{0}\right)\right)> \\
& \quad 0, i=1,2, \ldots, n,
\end{aligned}
$$

(II) $\varepsilon-(\bar{\nu}(T-\delta) /(\bar{\mu} T))>0$, where $\bar{\nu}=\max _{1 \leq i \leq n}\left\{\left|\nu_{i}\right|\right\}$, $\bar{\mu}=\min _{l \in \mathbb{S}} \mu_{l}$.

Proof. In Theorem 4, let $\alpha_{\ell i j}=\bar{\alpha}_{\ell i j}, \beta_{\ell i j}=\bar{\beta}_{\ell i j}, \xi_{\ell i j}=\bar{\xi}_{\ell i j}$, $\zeta_{\ell i j}=\bar{\zeta}_{\ell i j}, \epsilon_{\ell i j}=\bar{\epsilon}_{\ell i j}, \omega_{\ell i j}=\bar{\omega}_{\ell i j}$ for all $\ell=1,2, \ldots, p, i, j=$ $1,2, \ldots, n$; then $\lambda_{i}=\kappa_{i}$. Under condition (i), select $\rho_{i}=-v_{i}$, and Corollary 5 holds immediately from Theorem 4 .

In Theorem 4, we choose $\alpha_{\ell i j}=\bar{\alpha}_{\ell i j}=\beta_{\ell i j}=\bar{\beta}_{\ell i j}=\xi_{\ell i j}=$ $\bar{\xi}_{\ell i j}=\zeta_{\ell i j}=\bar{\zeta}_{\ell i j}=\eta^{*}=\epsilon_{\ell i j}=\bar{\epsilon}_{\ell i j}=\omega_{\ell i j}=\bar{\omega}_{\ell i j}=1 / p$ for $\ell=1,2, \ldots, p$, and $i, j=1,2, \ldots, n$; then

$$
\begin{aligned}
\tilde{\lambda}_{i}=\widetilde{\kappa}_{i} & \min _{l \in \mathbb{S}} \mu_{l}\left\{\sum_{k=1}^{m} \frac{4(p-1) \underline{D}_{i k}}{p \theta_{k}^{2}}+p a_{i}^{*}\right. \\
& -(p-1) \sum_{j=1}^{n}\left(\widetilde{b}_{i j} \widetilde{L}_{j}+\widetilde{c}_{i j} \widetilde{N}_{j}\right) \\
& -(p-1)(p-2) \sum_{j=1}^{n} \sigma_{i j} \\
& \left.-\sum_{j=1}^{n}\left(\widetilde{b}_{j i} \widetilde{L}_{j}+\widetilde{c}_{j i} \widetilde{N}_{j}+(p-1) \sigma_{j i}\right)\right\},
\end{aligned}
$$

$$
\begin{gathered}
\tilde{v}_{i}=\max _{l \in \mathbb{S}} \mu_{l}\left\{p k_{i i}+(p-1)\right. \\
\left.\times \sum_{\substack{j=1 \\
j \neq i}}^{n}\left|k_{i j}\right|+\sum_{\substack{j=1 \\
j \neq i}}^{n}\left|k_{j i}\right|\right\}, \\
\tilde{\eta}_{i}=\max _{l \in \mathbb{S}} \mu_{l} \sum_{j=1}^{n}\left[(p-1) \sigma_{j i}+\widetilde{c}_{j i} \widetilde{N}_{j}\right] .
\end{gathered}
$$

Then, as the proof of Theorem 4, we have the following.

Corollary 6. Under assumptions (H1)-(H3), the origin of system (3)-(5) under periodically intermittent control (13) is robust exponentially stable in pth moment if the following conditions hold:

(I) $\tilde{\lambda}_{i}-\widetilde{\nu}_{i}-\max _{l \in \mathbb{S}} \sum_{q=1}^{N} \gamma_{l q} \mu_{q}-\left(\widetilde{\eta}_{i} /\left(1-\tau_{0}\right)\right)>0, i=$ $1,2, \ldots, n$,

(II) there exists $\widetilde{\rho}_{i}>0$, such that $\tilde{\lambda}_{i}+\widetilde{\rho}_{i}-\max _{l \in \mathbb{S}} \sum_{q=1}^{N} \gamma_{l q} \mu_{q}-$ $\tilde{\eta}_{i} /\left(1-\tau_{0}\right)>0, i=1,2, \ldots, n$,

(III) $\widetilde{\varepsilon}-(\widetilde{\rho}(T-\delta) / \bar{\mu} T)>0$, where $\tilde{\rho}=\max _{1 \leq i \leq n} \widetilde{\rho}_{i}, \bar{\mu}=$ $\min _{l \in \mathbb{S}}\left\{\mu_{l}\right\}$.

Combining Corollary 5 and Corollary 6, we have the following.

Corollary 7. Under assumptions (H1)-(H3), the origin of system(3)-(5) under periodically intermittent control (13) is robust exponentially stable in pth moment if the following conditions hold:

$$
\begin{aligned}
\text { (I) } & \widetilde{v}_{i}<0, \widetilde{\lambda}_{i}-\widetilde{v}_{i}-\max _{l \in \mathbb{S}} \sum_{q=1}^{N} \gamma_{l q} \mu_{q}-\left(\widetilde{\eta}_{i} /\left(1-\tau_{0}\right)\right)>0, \\
& i=1,2, \ldots, n, \\
\text { (II) } & \widetilde{\varepsilon}-(\widehat{\nu}(T-\delta) / \bar{\mu} T)>0, \text { where } \widehat{\nu}=\max _{0 \leq i \leq n}\left\{\left|\widetilde{v}_{i}\right|\right\}, \bar{\mu}= \\
& \min _{l \in \mathbb{S}}\left\{\mu_{l}\right\} .
\end{aligned}
$$

Remark 8. By constructing an average Lyapunov function, the stabilization of stochastic Hopfield neural networks with distributed parameters was studied in [4]. The feedback controller in [4] was designed as the compound function of the state and activation function. Therefore, the feedback controller may be the nonlinear functions. However, we see in this paper that the control width is greater than the time delay and the periodically intermittent controller is linear and practical.

Remark 9. In [11-13, 24-26], robust exponential stability and exponential synchronization of some classes of neural networks with reaction-diffusion terms were discussed. The activation function satisfies Lipschitz condition. In fact, the activation function may be not monotone. But, from assumption (H1) in this paper, the activation functions include the monotone functions. So the results of this paper are less conservational and more general. 


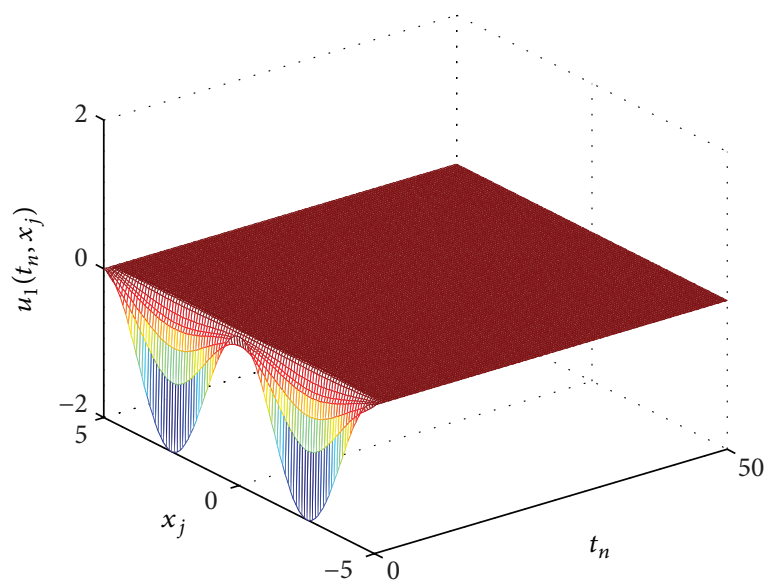

(a)

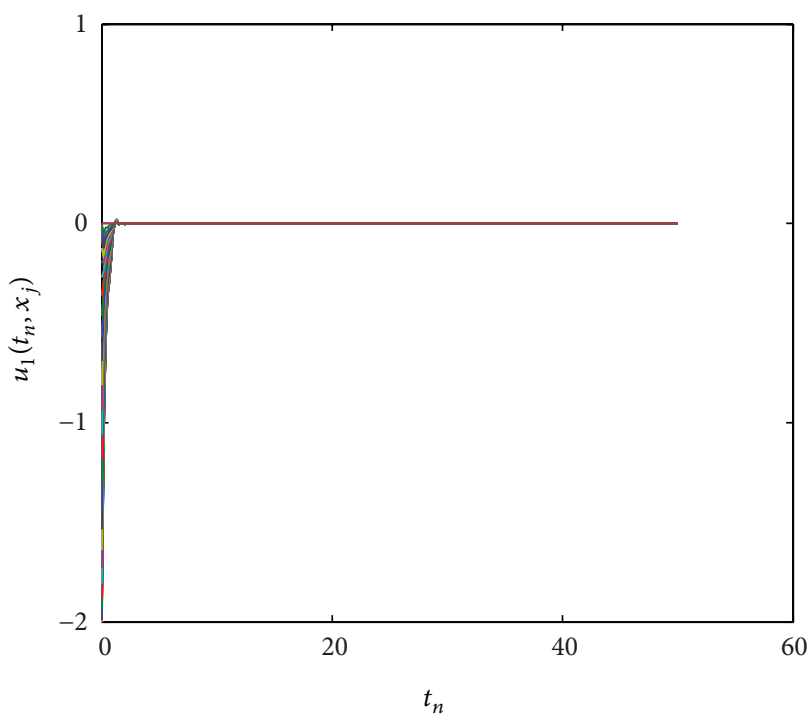

(c)

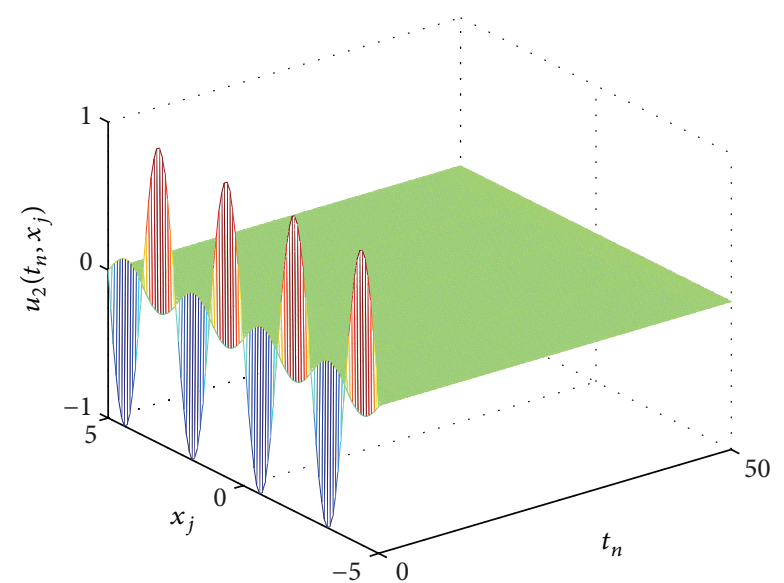

(b)

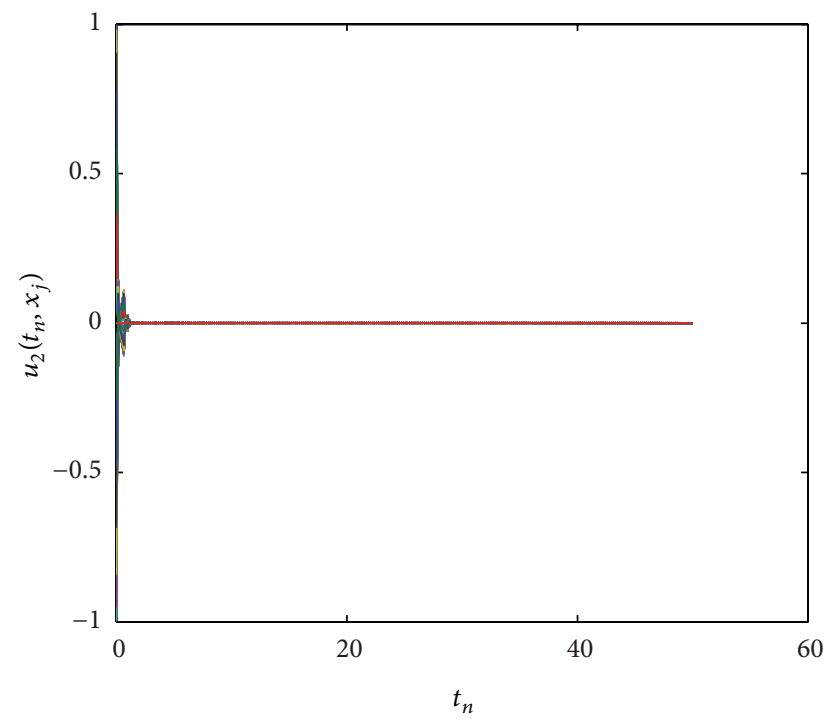

(d)

FIgURE 3: Surface curves and state trajectories for system (54) in model 1 under periodically intermittent control (13), $T=5$ and $\delta=4.7$.

Remark 10. In $[27,28]$, the periodically intermittent controller was designed to stabilization and synchronization of two classes of neural networks, where the activation functions satisfy

$$
0<\frac{f_{i}\left(s_{1}\right)-f_{i}\left(s_{2}\right)}{s_{1}-s_{2}} \leq L_{i}^{+}, \quad 0<\frac{g_{i}\left(s_{1}\right)-g_{i}\left(s_{2}\right)}{s_{1}-s_{2}} \leq N_{i}^{+} .
$$

In fact, they need $L_{i}^{+} \geq L_{i}^{-}>0, N_{i}^{+} \geq N_{i}^{-}>0$ in assumption (H1) of this paper. Obviously, the assumption (H1) of this paper is weaker than those of papers $[27,28]$.

Remark 11. In this paper, if the transmission delay $\tau_{i j}(t)$ is not continuous and differential, we can give new sufficient conditions ensuring robust exponential stabilization and antisynchronization for system (3)-(5) by applying linear matrix inequality (LMI) technique and periodically intermittent control. We will give the topics in future research.

\section{Numerical Example}

In this section, we give an example with numerical simulations to illustrate our result in the preceding section.

Example 1. Consider the 2-dimensional stochastic interval recurrent neural networks with two models as follows:

$$
\begin{aligned}
d u_{1}(t, x)=\{ & D_{1}(r(t)) \frac{\partial^{2} u_{1}(t, x)}{\partial x^{2}}-a_{1}(r(t)) u_{1}(t, x) \\
& +\sum_{j=1}^{2} b_{1 j}(r(t)) f_{j}\left(u_{j}(t, x)\right) \\
& \left.+\sum_{j=1}^{2} c_{1 j}(r(t)) g_{j}\left(u_{j}\left(t-\tau_{1 j}(t), x\right)\right)\right\} d t
\end{aligned}
$$




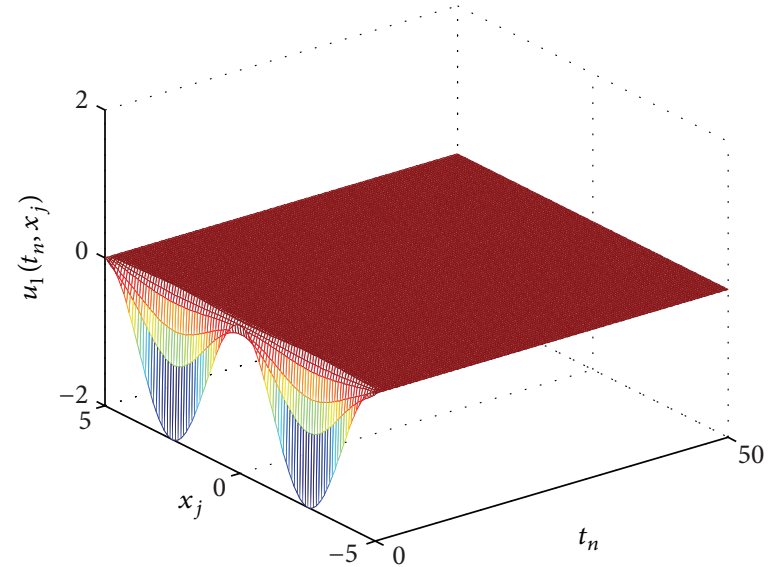

(a)

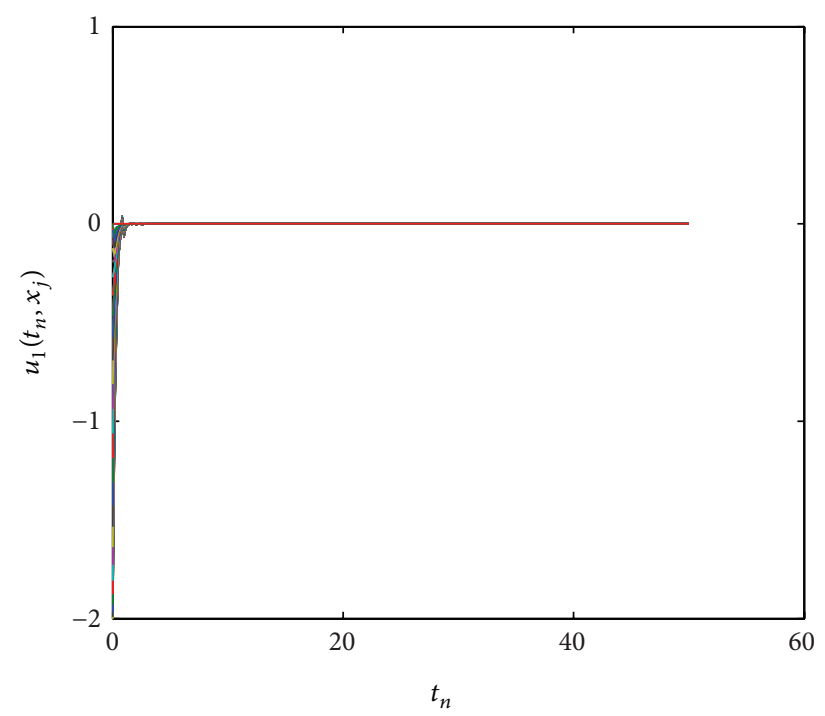

(c)

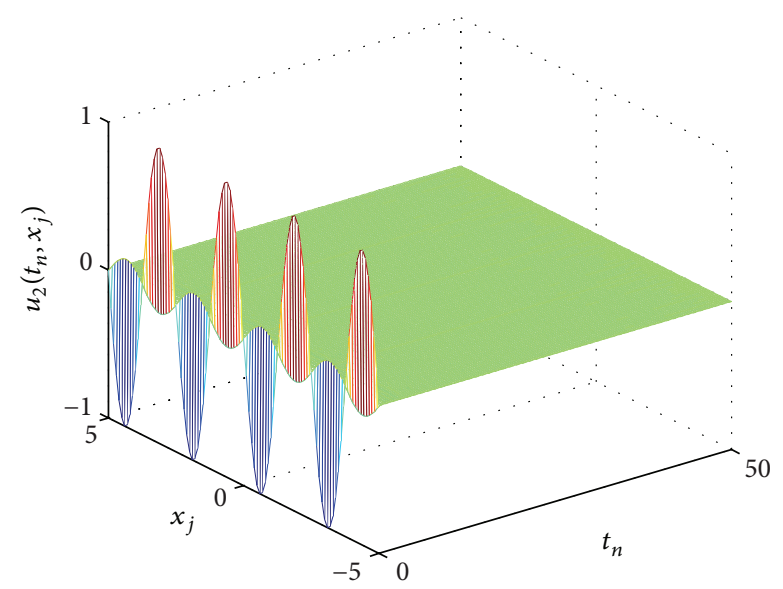

(b)

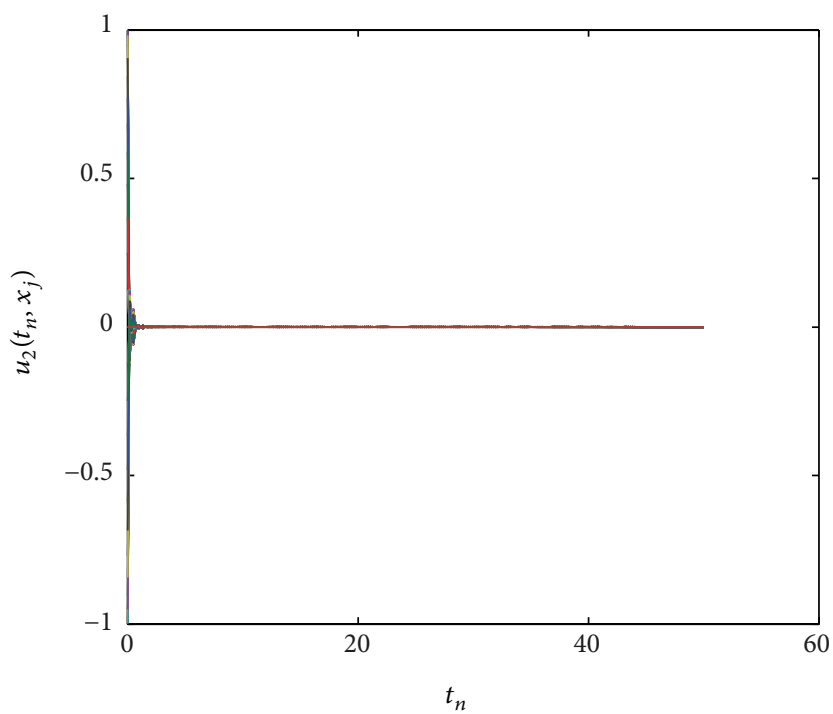

(d)

FIGURE 4: Surface curves and state trajectories for system (54) in model 2 under periodically intermittent control (13), $T=5$ and $\delta=4.7$.

$$
\begin{aligned}
&+\sum_{j=1}^{2} h_{1 j}\left(u_{j}(t, x), u_{j}\left(t-\tau_{1 j}(t), x\right)\right) d W_{j}(t), \\
& d u_{2}(t, x)=\left\{D_{2}(r(t)) \frac{\partial^{2} u_{2}(t, x)}{\partial x^{2}}-a_{2}(r(t)) u_{2}(t, x)\right. \\
&+\sum_{j=1}^{2} b_{2 j}(r(t)) f_{j}\left(u_{j}(t, x)\right)+\sum_{j=1}^{2} c_{2 j}(r(t)) \\
&\left.\quad \times g_{j}\left(u_{j}\left(t-\tau_{2 j}(t), x\right)\right)\right\} d t \\
&+\sum_{j=1}^{2} h_{2 j}\left(u_{j}(t, x), u_{j}\left(t-\tau_{2 j}(t), x\right)\right) d W_{j}(t),
\end{aligned}
$$

with the boundary conditions $u_{1}(t, 0)=u_{2}(t, 0)=$ $u_{1}(t, 2)=u_{2}(t, 2)=0, t \geq-1$ and the initial value $u_{1}(t, x)=e^{t}(\cos (2 \pi x)-1), u_{2}(t, x)=e^{2 t} \sin (4 \pi x), t \quad \epsilon$ $[-1,0] \times \Omega_{0}$, where $\tau_{i j}(t)=\left(e^{t} /\left(1+e^{t}\right)\right), \Omega_{0}=[-5,5] \in \mathbb{R}$, and the generator of the Markov chain

$$
\begin{gathered}
\Gamma=\left(\begin{array}{cc}
-1 & 1 \\
\frac{1}{2} & -\frac{1}{2}
\end{array}\right) \\
\left(u_{i}\right)=\frac{3}{4} \sin \left(u_{i}\right)+\frac{1}{4} u_{i} \\
\left(u_{i}\right)=\frac{1}{2}\left(\left|u_{i}+1\right|-\left|u_{i}-1\right|\right) \\
\left(u_{1}(t, x), u_{1}\left(t-\tau_{11}(t), x\right)\right) \\
=0.1 u_{1}(t, x)+0.2 u_{1}\left(t-\tau_{11}(t), x\right), \\
\left(u_{2}(t, x), u_{2}\left(t-\tau_{12}(t), x\right)\right) \\
=h_{21}\left(u_{1}(t, x), u_{1}\left(t-\tau_{21}(t)\right), x\right)=0,
\end{gathered}
$$$$
f_{i}\left(u_{i}\right)=\frac{3}{4} \sin \left(u_{i}\right)+\frac{1}{4} u_{i}
$$$$
g_{i}\left(u_{i}\right)=\frac{1}{2}\left(\left|u_{i}+1\right|-\left|u_{i}-1\right|\right),
$$$$
h_{11}\left(u_{1}(t, x), u_{1}\left(t-\tau_{11}(t), x\right)\right)
$$$$
h_{12}\left(u_{2}(t, x), u_{2}\left(t-\tau_{12}(t), x\right)\right)
$$ 


$$
\begin{aligned}
h_{22} & \left(u_{2}(t, x), u_{2}\left(t-\tau_{22}(t), x\right)\right) \\
= & 0.3 u_{2}(t, x)+0.4 u_{2}\left(t-\tau_{22}(t), x\right) .
\end{aligned}
$$

We assume that the interval matrices are the same in every model; let

$$
\begin{aligned}
& D(r(t))=\left(\begin{array}{ll}
D_{1}(r(t)) & \\
& D_{2}(r(t))
\end{array}\right)=\left(\begin{array}{ll}
{[1,2]} & \\
& {[2,3]}
\end{array}\right), \\
& A(r(t))=\left(\begin{array}{ll}
a_{1}(r(t)) & \\
& a_{2}(r(t))
\end{array}\right) \\
& =\left(\begin{array}{ll}
{[0.28,0.42]} & \\
& {[0.18,0.35]}
\end{array}\right) \text {, } \\
& B(r(t))=\left(\begin{array}{lll}
B_{11}(r(t)) & B_{12}(r(t)) \\
B_{21}(r(t)) & B_{22}(r(t))
\end{array}\right)=\left(\begin{array}{ll}
{[4,5]} & \\
& {[3,4]}
\end{array}\right), \\
& C(r(t))=\left(\begin{array}{ll}
C_{11}(r(t)) & C_{12}(r(t)) \\
C_{21}(r(t)) & C_{22}(r(t))
\end{array}\right) \\
& =\left(\begin{array}{ll}
{[0.2,0.3]} & \\
& {[0.3,0.4]}
\end{array}\right) .
\end{aligned}
$$

The surface curves and state trajectories of system (54) in model (1) and model (2) are given, respectively, as shown in Figures 1 and 2 . They exhibit instability behavior.

Let $p=2, \mu_{1}=2, \mu_{2}=4$. By simple calculation, we obtain

$$
\begin{array}{ll}
L_{i}^{-}=-\frac{1}{2}, \quad L_{i}^{+}=1, & N_{i}^{-}=0, \\
N_{i}^{+}=1, \quad \tau=1, & \tau_{0}=\frac{1}{4} \\
\tilde{\lambda}_{1}=\tilde{\kappa}_{1}=-40.16, & \tilde{\eta}_{1}=1.52 \\
\tilde{\lambda}_{2}=\tilde{\kappa}_{2}=-34.4, \quad \tilde{\eta}_{2}=2.88 .
\end{array}
$$

Now, we consider the periodically intermittent control (13), where the parameters are given as follows:

$$
k_{11}=-10, \quad k_{22}=-10, \quad k_{12}=0, \quad k_{21}=0 .
$$

Then $\widetilde{\nu}_{1}=-80$ and $\widetilde{v}_{2}=-80$,

$$
\begin{aligned}
& \tilde{\lambda}_{1}-\widetilde{\nu}_{1}-\max _{l \in \mathbb{S}} \sum_{q=1}^{N} \gamma_{l q} \mu_{q}-\frac{\tilde{\eta}_{1}}{1-\tau_{0}}>0, \\
& \tilde{\lambda}_{2}-\widetilde{\nu}_{2}-\max _{l \in \mathbb{S}} \sum_{q=1}^{N} \gamma_{l q} \mu_{q}-\frac{\tilde{\eta}_{2}}{1-\tau_{0}}>0 .
\end{aligned}
$$

From $H_{i}\left(\widetilde{\varepsilon}_{i}\right)=\tilde{\lambda}_{i}-\widetilde{\nu}_{i}-\max _{l \in \mathbb{S}} \sum_{q=1}^{N} \gamma_{l q} \mu_{q}-\widetilde{\varepsilon}_{i} \max _{l \in \mathbb{S}} \mu_{l}-$ $\left(\widetilde{\eta}_{i} /\left(1-\tau_{0}\right)\right) e^{\widetilde{\varepsilon}_{i} \tau}=0, i=1,2$, we have

$$
\widetilde{\varepsilon}=\min \left\{\widetilde{\varepsilon}_{1}, \widetilde{\varepsilon}_{2}\right\}=\min \{2.605,2.413\}=2.413 .
$$

Choose $T=5$, from Corollary $7, \delta=4.7$. Then the origin of system (54) under the periodically intermittent controller (13) is robust exponentially stable in mean square. The surface curves and state trajectories in model (1) and model (2) are given, respectively, as shown in Figures 3 and 4 .

\section{Conflict of Interests}

The authors declare no competing financial interests.

\section{Acknowledgment}

This work was partially supported by the National Natural Science Foundation of China under Grants nos. 61374085 and 61304068 and the Fundamental Research Funds for the Central Universities under Grants nos. $2013 Z Z 0056$ and 2013 QC019.

\section{References}

[1] X. X. Liao and X. Mao, "Stability of stochastic neural networks," Neural, Parallel \& Scientific Computations, vol. 4, no. 2, pp. 205224, 1996.

[2] X. X. Liao and X. Mao, "Exponential stability and instability of stochastic neural networks," Stochastic Analysis and Applications, vol. 14, no. 2, pp. 165-185, 1996.

[3] S. Blythe, X. Mao, and A. Shah, "Razumikhin-type theorems on stability of stochastic neural networks with delays," Stochastic Analysis and Applications, vol. 19, no. 1, pp. 85-101, 2001.

[4] Q. Luo, F. Deng, J. Bao, B. Zhao, and Y. Fu, "Stabilization of stochastic Hopfield neural network with distributed parameters," Science in China F, vol. 47, no. 6, pp. 752-762, 2004.

[5] Q. Luo, F. Deng, X. Mao, J. Bao, and Y. Zhang, "Theory and application of stability for stochastic reaction diffusion systems," Science in China F, vol. 51, no. 2, pp. 158-170, 2008.

[6] X. Liao, Y. Fu, J. Gao, and X. Zhao, "Stability of Hopfield neural networks with reaction-diffusion terms," Acta Electronica Sinica, vol. 28, no. 1, pp. 78-80, 2000.

[7] X. Liao, S. Yang, S. Cheng, and Y. Fu, "Stability of general neural networks with reaction-diffusion," Science in China F, vol. 44, no. 5, pp. 389-395, 2001.

[8] L. Wang and D. Xu, "Global exponential stability of Hopfield reaction-diffusion neural networks with time-varying delays," Science in China F, vol. 46, no. 6, pp. 466-474, 2003.

[9] Q. Zhu, X. Li, and X. Yang, "Exponential stability for stochastic reaction-diffusion BAM neural networks with time-varying and distributed delays," Applied Mathematics and Computation, vol. 217, no. 13, pp. 6078-6091, 2011.

[10] C. Hu, H. Jiang, and Z. Teng, "Impulsive control and synchronization for delayed neural networks with reaction-diffusion terms," IEEE Transactions on Neural Networks, vol. 21, no. 1, pp. 67-81, 2010.

[11] X. Xu, J. Zhang, and W. Zhang, "Stochastic exponential robust stability of interval neural networks with reaction-diffusion terms and mixed delays," Communications in Nonlinear Science and Numerical Simulation, vol. 17, no. 12, pp. 4780-4791, 2012.

[12] L. Wang and Y. Gao, "Global exponential robust stability of reaction-diffusion interval neural networks with time-varying delays," Physics Letters A, vol. 350, no. 5-6, pp. 342-348, 2006.

[13] J. G. Lu, "Robust global exponential stability for interval reaction-diffusion Hopfield neural networks with distributed delays," IEEE Transactions on Circuits and Systems II: Express Briefs, vol. 54, no. 12, pp. 1115-1119, 2007.

[14] Y. Shen and J. Wang, "Noise-induced stabilization of the recurrent neural networks with mixed time-varying delays and Markovian-switching parameters," IEEE Transactions on Neural Networks, vol. 18, no. 6, pp. 1857-1862, 2007. 
[15] Y. Shen and J. Wang, "Almost sure exponential stability of recurrent neural networks with Markovian switching," IEEE Transactions on Neural Networks, vol. 20, no. 5, pp. 840-855, 2009.

[16] Q. Zhu and J. Cao, "Robust exponential stability of markovian jump impulsive stochastic Cohen-Grossberg neural networks with mixed time delays," IEEE Transactions on Neural Networks, vol. 21, no. 8, pp. 1314-1325, 2010.

[17] Q. Zhu and J. Cao, "Exponential stability of stochastic neural networks with both Markovian jump parameters and mixed time delays," IEEE Transactions on Systems, Man, and Cybernetics B: Cybernetics, vol. 41, no. 2, pp. 341-353, 2011.

[18] Q. Zhu and J. Cao, "Stability analysis of Markovian jump stochastic BAM neural networks with impulse control and mixed time delays," IEEE Transactions on Neural Networks and Learning Systems, vol. 23, no. 3, pp. 467-479, 2012.

[19] Q. Zhu and J. Cao, "Stability of Markovian jump neural networks with impulse control and time varying delays," Nonlinear Analysis: Real World Applications, vol. 13, no. 5, pp. 2259-2270, 2012.

[20] Q. Zhu and J. Cao, "pth moment exponential synchronization for stochastic delayed Cohen-Grossberg neural networks with Markovian switching," Nonlinear Dynamics, vol. 67, no. 1, pp. 829-845, 2012.

[21] H. Huang, D. W. C. Ho, and Y. Qu, "Robust stability of stochastic delayed additive neural networks with Markovian switching," Neural Networks, vol. 20, no. 7, pp. 799-809, 2007.

[22] X. Mao, "Exponential stability of stochastic delay interval sysems with Markovian switching," IEEE Transactions on Automatic Control, vol. 47, no. 10, pp. 1604-1612, 2002.

[23] Z.-H. Guan and H. Zhang, "Stabilization of complex network with hybrid impulsive and switching control," Chaos, Solitons \& Fractals, vol. 37, no. 5, pp. 1372-1382, 2008.

[24] Q. Gan, "Exponential synchronization of stochastic CohenGrossberg neural networks with mixed time-varying delays and reaction-diffusion via periodically intermittent control," Neural Networks, vol. 31, pp. 12-21, 2012.

[25] Q. Gan, "Exponential synchronization of stochastic neural networks with leakage delay and reaction-diffusion terms via periodically intermittent control," Chaos, vol. 22, no. 1, Article ID 013124, 2012.

[26] Q. Gan, "Exponential synchronization of stachastic fuzzy cellular neural networks with reaction-diffusion terms via periodically intermittent control," Neural Processing Letters, vol. 37, pp. 393-410, 2013.

[27] C. Hu, J. Yu, H. Jiang, and Z. Teng, "Exponential lag synchronization for neural networks with mixed delays via periodically intermittent control," Chaos, vol. 20, no. 2, Article ID 023108, 2010.

[28] C. Hu, J. Yu, H. Jiang, and Z. Teng, "Exponential stabilization and synchronization of neural networks with time-varying delays via periodically intermittent control," Nonlinearity, vol. 23, no. 10, pp. 2369-2391, 2010.

[29] J. Huang, C. Li, and Q. Han, "Stabilization of delayed chaotic neural networks by periodically intermittent control," Circuits, Systems, and Signal Processing, vol. 28, no. 4, pp. 567-579, 2009.

[30] G. da Prato and J. Zabczyk, Stochastic Equations in Infinite Dimensions, vol. 44 of Encyclopedia of Mathematics and Its Applications, Cambridge University Press, Cambridge, Mass, USA, 1992.
[31] X. Mao and C. Yuan, Stochastic Differential Equations with Markovian Switching, Imperial College Press, London, UK, 2006.

[32] C.-J. Cheng, T.-L. Liao, J.-J. Yan, and C.-C. Hwang, "Exponential synchronization of a class of neural networks with time-varying delays," IEEE Transactions on Systems, Man, and Cybernetics B: Cybernetics, vol. 36, no. 1, pp. 209-215, 2006.

[33] H. Lu, "Chaos attractors in delayed neural networks," Physics Letters A, vol. 298, pp. 109-116, 2002. 


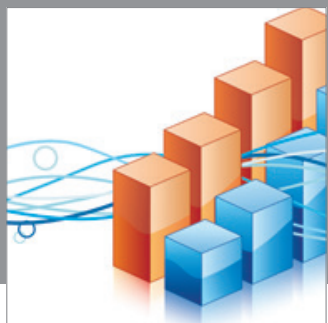

Advances in

Operations Research

mansans

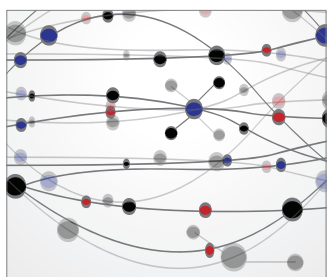

The Scientific World Journal
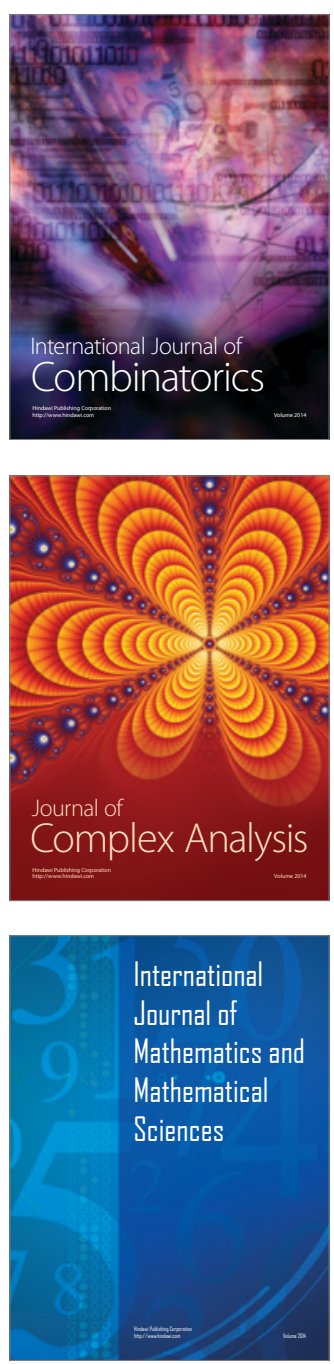
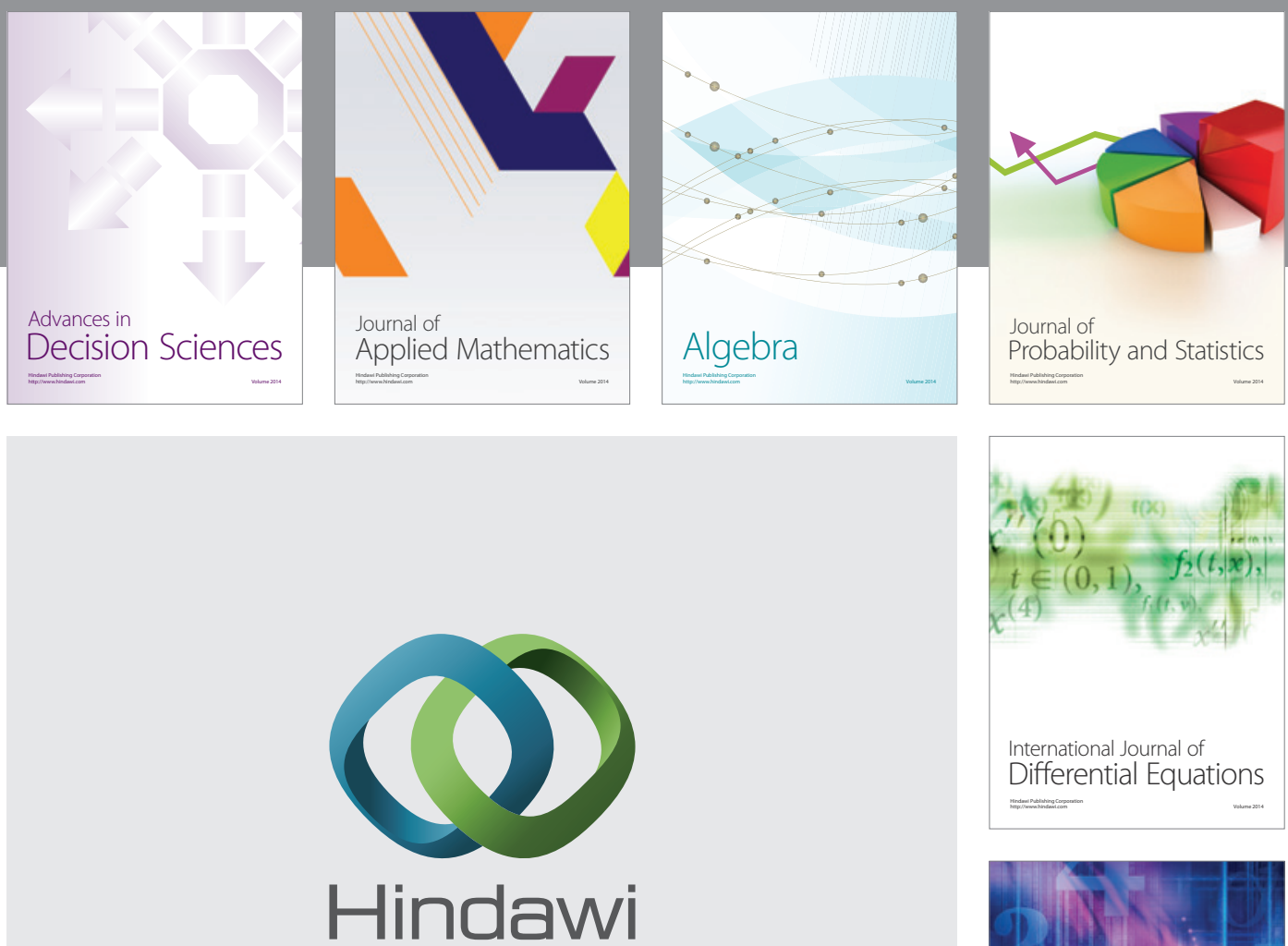

Submit your manuscripts at http://www.hindawi.com
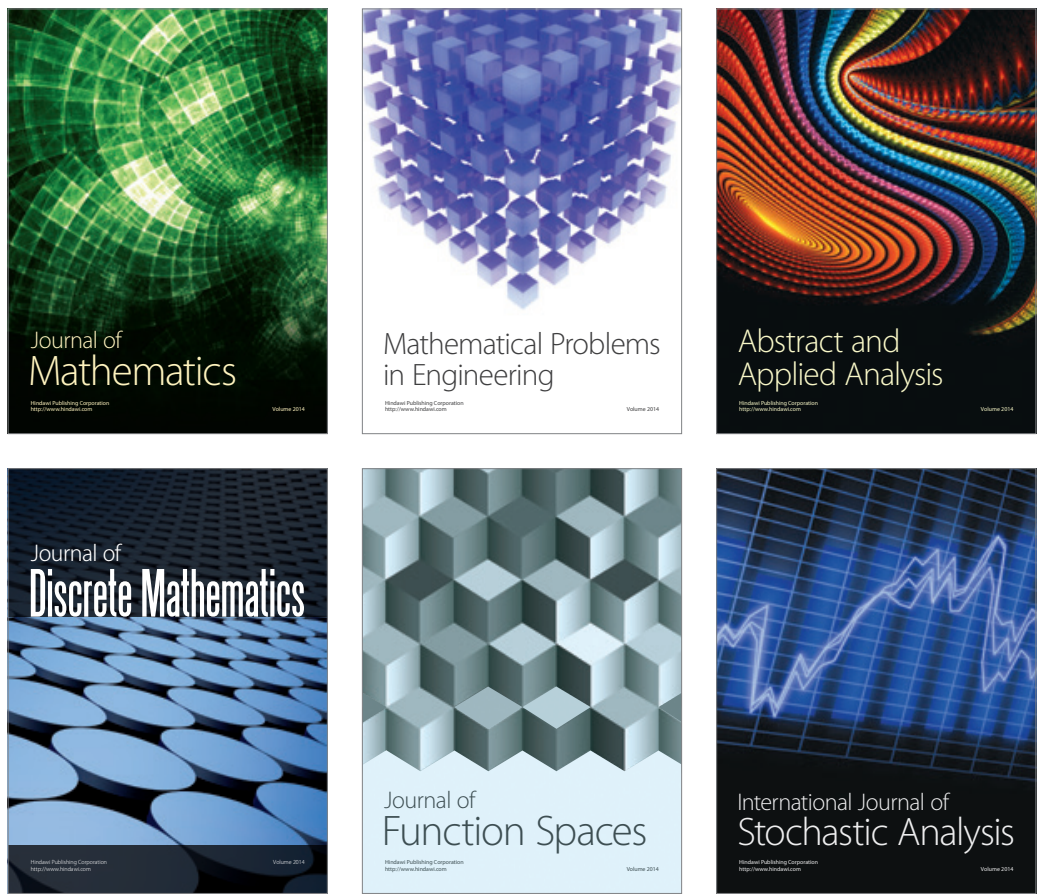

Journal of

Function Spaces

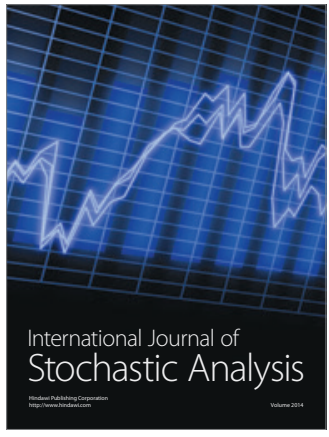

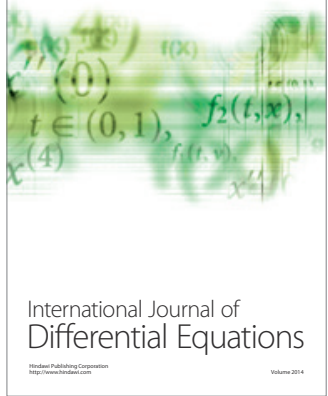
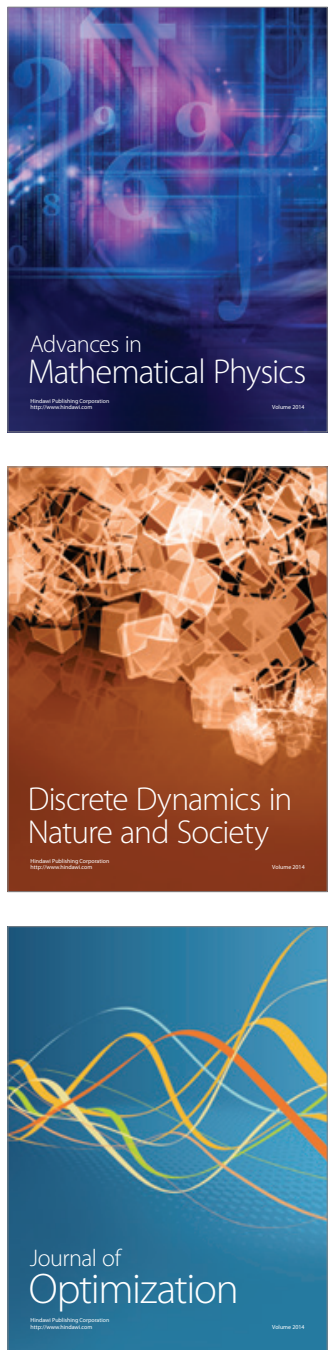